\title{
The dihadron fragmentation function and its evolution
}

\author{
A. Majumder and Xin-Nian Wang \\ Nuclear Science Division, \\ Lawrence Berkeley National Laboratory \\ 1 Cyclotron road, Berkeley, CA 94720
}

(Dated: April 27, 2006)

\begin{abstract}
Dihadron fragmentation functions and their evolution are studied in the process of $e^{+} e^{-}$annihilation. Under the collinear factorization approximation and facilitated by the cut-vertex technique, the two hadron inclusive cross section at leading order (LO) is shown to factorize into a short distance parton cross section and a long distance dihadron fragmentation function. We provide the definition of such a dihadron fragmentation function in terms of parton matrix elements and derive its DGLAP evolution equation at leading log. The evolution equation for the non-singlet quark fragmentation function is solved numerically with a simple ansatz for the initial condition and results are presented for cases of physical interest.
\end{abstract}

PACS numbers: 13.66.Bc, 25.75.Gz, 11.15.Bt

\section{INTRODUCTION}

Lattice QCD calculations 1 predict a phase transition from a hadronic gas to a quark gluon plasma (QGP) at very high energy densities in which quarks and gluons are no longer confined to the size of individual hadrons. To create such a dense and hot matter, heavy ions are accelerated to extremely high energies to collide with each other. If formed in such heavy-ion collisions, the QGP is rather short lived and hadronizes quickly into a plethora of mesons and baryons. Hence, the existence of such a state in the history of a given collision must be surmised through a variety of indirect probes. One of the most promising signatures has been that of jet quenching $\boldsymbol{2}$, which leads to the suppression of high $p_{T}$ particles emanating from such collisions. Such jet quenching phenomena have been among the most striking experimental discoveries from the Relativistic Heavy Ion Collider (RHIC) at Brookhaven National Laboratory. Not only has the suppression of single inclusive high $p_{T}$ hadron spectra been observed ?, but also the disappearance of back-to-back correlations of high $p_{T}$ hadrons has been noted 4 . Both phenomena and the observed azimuthal anisotropy of high $p_{T}$ hadron spectra are qualitatively consistent with the picture of parton energy loss that leads to jet quenching. This is indicative of the formation of a hot medium which is opaque to energetic partons and has a parton density about 30 times higher than in a cold heavy nucleus.

In the investigation of jet suppression, correlations between two high $p_{T}$ hadrons in azimuthal angle are used to study the change of jet structure 4 . While the back-toback correlations are suppressed in central $A u+A u$ collisions, indicating parton energy loss, the same-side correlations remain approximately the same as in $p+p$ and $d+A u$ collisions. Given the experimental kinematics, this is considered as an indication of parton hadronization outside the medium. However, since the same-side correlation corresponds to two-hadron distribution within a single jet, the observed phenomenon is highly nontriv- ial. To answer the question as to why a parton with a reduced energy would give the same two-hadron distribution, one has to take a closer look at the single and double hadron fragmentation functions and their modification in medium. In this paper we take the first step by studying the dihadron fragmentation functions in the process of $e^{+} e^{-}$annihilations.

Inclusive hadron production cross sections in $e^{+} e^{-}$collisions have turned out to be one of the many successful predictions of perturbative QCD $5,6,7$. For reactions at an energy scale much above $\Lambda_{Q C D}$ one can factorize the cross section into a short-distance parton cross section which is computable order by order as a series in $\alpha_{s}\left(Q^{2}\right)$; and a long-distance phenomenological object (the single hadron inclusive fragmentation function) which contains the non-perturbative information of parton hadronization 8 . These fragmentation functions can be defined in an operator formalism $\mathrm{C}$ and hence are valid beyond the perturbative theory. They, however, cannot be calculated perturbatively and have to be, instead, inferred from experiments. The definition of these functions affords them the mantle of being universal or process-independent. Once measured in one process, e.g. $e^{+} e^{-}$annihilation, they can be applied to another, e.g. deep inelastic scattering or $p+p$ collisions, and therein lies the predictive power. Another contribution of pQCD rests in the fact that once these functions are measured at a given energy scale, they can be predicted for all other energy scales via the Dokshitzer-Gribov-Lipatov-Altarelli-Parisi (DGLAP) evolution equations 10, 11, 12 .

The single inclusive fragmentation function $D_{q}^{h}\left(z, Q^{2}\right)$ can be interpreted as a multiplicity distribution for hadrons of type $h$ with a longitudinal momentum fraction $z$ that materialize from a fragmenting parton of flavour $q$. One can have, in principle, an $n$-hadron fragmentation function $D_{q}^{h_{1}, h_{2}, \ldots h_{n}}\left(z_{1}, z_{2}, \ldots z_{n}, Q^{2}\right)$ which counts the number of hadrons of type $h_{1}, h_{2}, \ldots h_{n}$ with momentum fractions $z_{1}, z_{2}, \ldots z_{n}$ materializing from a fragmenting parton $q$. In this article, we will be con- 
cerned with the double inclusive fragmentation function $D_{q}^{h_{1}, h_{2}}\left(z_{1}, z_{2}, Q^{2}\right)$ or the dihadron fragmentation function. The operator definition of this function is not merely a trivial extension of the single hadron case; there are no straightforward sum rules connecting it to single inclusive fragmentation functions. Similar functions have been considered previously 13, 14,15 . However, such formulations considered the double fragmentation function with a fixed angle $\delta$ (or a fixed tranverse momentum difference $q_{T}$ ) between the two hadrons. For a large enough choice of $\delta$ (or $q_{T}$ ) the dominant contribution to $D_{q}^{h_{1}, h_{2}}\left(z_{1}, z_{2}, \delta, Q^{2}\right)$ was postulated to emanate from a process where the fragmenting parton had undergone a split into two partons which then fragmented independently. This formulation, however, requires $\delta$ (or $q_{T}$ ) to be large enough that the splitting process may be calculated in perturbation theory. In our formulation, the internal angle $\delta$ (or relative momentum $q_{T}$ ) will be explicitly integrated over. In this regard, our calculations are similar in spirit to those of Ref. 16. In that effort, general evolution equations for $n$-hadron fragmentation functions were motivated, and an algebraic solution of the moments of the fragmentation functions was obtained. In none of the previous studies, however, has an attempt been made to generalize the operator definition of the fragmentation function from one hadron to many hadrons. Evaluation of the $n$-hadron production cross section at leading order (LO) and leading twist allows one to analytically define such a function. Evaluation of corrections to the same cross section at leading log (LL) and leading twist allows one to derive the evolution equations for such functions. This is the objective of the current study. To the knowledge of the authors, no such calculation has hitherto been attempted.

Our eventual goal is to derive the medium modification to the dihadron fragmentation function as the fragmenting parton propagates through the medium. As shown in the case of single hadron fragmentation functions 17 , the medium modification of the fragmentation function due to multiple scattering and induced gluon radiation resembles very much that of radiative corrections due to gluon bremsstrahlung in vacuum. Therefore, the study of DGLAP evolution of dihadron fragmentation functions in this paper can already provide us hints of what one may expect for medium modifications.

The remaining sections are organized as follows: in Sec. II we present a general discussion of the double fragmentation function. We outline how such a function may be isolated in the expression for a double differential inclusive cross section and discuss the possible nature of its evolution equations. In Sec. III we begin with the S-matrix expression for the double differential cross section for the production of two hadrons in $e^{+} e^{-}$collisions at leading order (LO); we then factorize the expression at leading twist into the conventional hard part and the double fragmentation function. We also derive the rules for such an object in the cut-vertex technique of Mueller. In Sec. IV we write down the double differential cross section at next-to-leading order (NLO) and once again factorize it at leading twist into the conventional hard part and NLO correction to the fragmentation function. Using these we derive the DGLAP $10,11,12$ evolution equations for the double fragmentation functions. In Sec. $\mathrm{V}$ we focus on the evolution equation for the non-singlet (NS) quark fragmentation function and solve its evolution equation numerically. Finally in Sec. VI we discuss the results of our calculation and present our conclusions.

\section{THE PARTON MODEL}

In this section, we present a general discussion of the properties of a dihadron fragmentation function within a parton-model-like picture with collinear factorization. However, all our assumptions will be demonstrated to hold explicitly in an operator formalism at leading log and leading twist in the subsequent sections.

We consider the following semi-inclusive process

$$
e^{+}+e^{-} \rightarrow \gamma^{*} \rightarrow h_{1}+h_{2}+X
$$

of $e^{+} e^{-}$annihilation. We consider two-jet events where both the identified hadrons $h_{1}$ and $h_{2}$ emanate from the same jet. At leading order in the strong coupling this occurs from the conversion of the virtual photon into a back-to-back quark and antiquark pair which fragment into two jets of hadrons.

In this scenario, at a large $Q^{2}$ of the reaction, one may factorize the cross section of single inclusive hadron production as 18

$$
\frac{d \sigma}{d z}=\sum_{q} \sigma_{0}^{q \bar{q}}\left[D_{q}^{h}(z)+D_{\bar{q}}^{h}(z)\right]
$$

with $D_{q}^{h}(z)$ and $D_{\bar{q}}^{h}(z)$ as the single inclusive quark fragmentation functions. The total cross section for the annihilation of an electron positron pair to a quark and an anti-quark, $\sigma_{0}^{q \bar{q}}$ at leading order is

$$
\sigma_{0}^{q \bar{q}}=e_{q}^{2} N_{c} \frac{4 \pi \alpha^{2}}{3 s}
$$

Here, $\epsilon_{q}$ is the fractional charge of the quark in units of an electron charge, $s$ is the square of center of mass energy of the $e^{+} e^{-}$pair, and $N_{c}=3$ is the number of colors in the fundamental representation of QCD. The fractional momentum $z$ represents the the light-cone momentum fraction of the hadrons to the parent partons, i.e.,

$$
z=\frac{\mathbf{p}_{h} \cdot \mathbf{n}}{\mathbf{p} \cdot \mathbf{n}},
$$

where we use the notation of bold face letters representing four-vectors. The lightlike four-vector $\mathbf{n} \equiv$ $\left[n^{+}, n^{-}, n_{\perp}\right]=[0,1,0]$ is taken conjugate to a given momentum, as yet unspecified. 
Similarly, one can expect to obtain the two-hadron inclusive cross section from Eq. II by replacing the single inclusive fragmentation functions $D_{q}^{h}\left(z_{1}\right)$ with the double inclusive functions $D_{q}^{h_{1} h_{2}}\left(z_{1}, z_{2}\right)$,

$$
\frac{d^{2} \sigma}{d z_{1} d z_{2}}=\sum_{q} \sigma_{0}^{q \bar{q}}\left[D_{q}^{h_{1} h_{2}}\left(z_{1}, z_{2}\right)+D_{\bar{q}}^{h_{1} h_{2}}\left(z_{1}, z_{2}\right)\right]
$$

We will not discuss cases in which each of the two hadrons emerges independently from each of the back-to-back quark and antiquark jets.

In the parton model 18 , at NLO with a single gluon radiative correction, the two hadron inclusive cross section in $e^{+} e^{-}$annihilation can be expressed as a convolution of the fragmentation functions with the differential partonic cross sections (see Sec. 3.3 of Ref. 18),

$$
\begin{aligned}
d^{2} \sigma\left(z_{1}, z_{2}, Q^{2}\right)= & \left(\frac{d \sigma_{q}}{d y}\right) d y D_{q}^{h_{1}, h_{2}}\left(x_{1}, x_{2}\right) d x_{1} d x_{2} \\
+ & \left(\frac{d \sigma_{\bar{q}}}{d y}\right) d y D_{\bar{q}}^{h_{1}, h_{2}}\left(x_{1}, x_{2}\right) d x_{1} d x_{2} \\
+ & \left(\frac{d \sigma_{g}}{d y}\right) d y D_{g}^{h_{1}, h_{2}}\left(x_{1}, x_{2}\right) d x_{1} d x_{2} \\
+ & \left(\frac{d \sigma_{g q}}{d y}\right) d y\left[D_{g}^{h_{1}}\left(x_{1}\right) D_{q}^{h_{2}}\left(x_{2}\right)+\right. \\
& \left.\left(h_{1}, z_{1}\right) \rightarrow\left(h_{2}, z_{2}\right)\right] d x_{1} d x_{2} \\
+ & \left(\frac{d \sigma_{g \bar{q}}}{d y}\right) d y\left[D_{g}^{h_{1}}\left(x_{1}\right) D_{\bar{q}}^{h_{2}}\left(x_{2}\right)+\right. \\
& \left.\left(h_{1}, z_{1}\right) \rightarrow\left(h_{2}, z_{2}\right)\right] d x_{1} d x_{2} .
\end{aligned}
$$

There are two distinct types of contributions in the above equation. The first one is determined by the two-hadron fragmentation functions of single partons that have the pQCD differential cross sections, $d \sigma_{q} / d y$, $d \sigma_{\bar{q}} / d y, d \sigma_{g} / d y$, and momentum fraction $y$. The second contribution corresponds to two, almost collinear, partons (a gluon and a quark or antiquark with pQCD cross sections $d \sigma_{g q} / d y$ and $d \sigma_{g \bar{q}} / d y$ ) splitting from the same parent parton and then fragmenting independently into hadrons. In this case, the quark (antiquark) carries momentum fraction $y$ and the gluon carries $1-y$. One of the identified hadrons comes from each of these partons.

The two hadrons $h_{1}$ and $h_{2}$ have momentum fractions $x_{1}$ and $x_{2}$ of their immediate parent parton which itself is endowed with a momemtum fraction $y$ or $1-y$. Relating the inside parton variables $x_{1}, x_{2}, y$ to the outside hadron variables we obtain

$$
x_{1}=z_{1} / y, x_{2}=z_{2} / y
$$

for the first three terms and

$$
x_{1}=z_{1} / y, x_{2}=z_{2} /(1-y)
$$

for the last two terms. Using the pQCD partonic cross sections in the massive gluon scheme 18 , one can obtain the double differential cross section for the production of two hadrons with momentum fractions $z_{1}, z_{2}$ as,

$$
\begin{aligned}
\frac{d^{2} \sigma}{d z_{1} d z_{2}} & =\sum_{q} \sigma_{0}^{q \bar{q}}\left[\int _ { z _ { 1 } + z _ { 2 } } ^ { 1 } \frac { d y } { y ^ { 2 } } \left\{\left(1+\frac{\alpha_{s}}{\pi}\right) \delta(1-y)\right.\right. \\
& +\frac{\alpha_{s}}{2 \pi} P_{q \rightarrow q g}(y) \log \left(Q^{2} / m_{g}^{2}\right)+\alpha_{s} f_{q}^{\left.e^{+} e^{-}(y)\right\}} \\
& \times\left\{D_{q}^{h_{1}, h_{2}}\left(z_{1} / y, z_{2} / y\right)+D_{\bar{q}}^{h_{1}, h_{2}}\left(z_{1} / y, z_{2} / y\right)\right\} \\
& +2\left\{\frac{\alpha_{s}}{2 \pi} P_{q \rightarrow g q}(y) \log \left(Q^{2} / m_{g}^{2}\right)+\alpha_{s} f_{g}^{e^{+} e^{-}}(y)\right\} \\
& \times D_{g}^{h_{1}, h_{2}}\left(z_{1} / y, z_{2} / y\right)+\int_{z_{1}}^{1-z_{2}} \frac{d y}{y(1-y)} \\
& \times \frac{\alpha_{s}}{2 \pi} \hat{P}_{q \rightarrow q g}(y) \log \left(Q^{2} / m_{g}^{2}\right) \\
& \times\left\{D_{q}^{h_{1}}\left(z_{1} / y\right) D_{g}^{h_{2}}\left(z_{2} /(1-y)\right)\right. \\
& \left.+D_{\bar{q}}^{h_{1}}\left(z_{1} / y\right) D_{g}^{h_{2}}\left(z_{2} /(1-y)\right)\right\}+1 \rightarrow 2 .
\end{aligned}
$$

Here the switch between the indices $1 \rightarrow 2$ is only meant for the last $y$ integration. The $P(y)$ functions are the regular splitting functions which contain both the real and virtual contributions and thus have no infrared divergences. The $\hat{P}(y)$ functions contain no contributions from virtual diagrams. The $f(y)$ 's are the scheme dependent functions obtained in the massive gluon scheme, where $m_{g}$ is the fictitious gluon mass introduced to regulate the collinear divergences. In the subsequent discussion we will focus on the leading $\log$ (LL) piece of the above expression. Thus one can drop the scheme dependent $f$ functions.

Note that up to this point we have simply retraced the sequence of steps in the evaluation of the radiative corrections to the single inclusive fragmentation functions in the parton model. What is new in the case of two hadron inclusive cross section is the contribution from the splitting into a quark and gluon followed by independent fragmentation. The $\log \left(Q^{2} / m_{g}^{2}\right)$ in this contribution originates from an integration over the transverse momentum $q_{\perp}$ of the quark and gluon emanating from the split. For very small values of $q_{\perp}$, other higher order and nonperturbative processes become important that will invalidate the picture of independent fragmentation of the two partons. For $q_{\perp} \gg \Lambda_{Q C D}$, however, the higher order corrections will be suppressed and the quark and gluon will fragment incoherently in LL approximation (this was first pointed out in Ref. 15). A simple proof of this statement has been included in the Appendix. In this paper, we introduce a cut-off scale $\mu_{\perp}$ that separates two regimes of two-parton fragmentation according to the value of $q_{\perp}$ : independent fragmentation for $q_{\perp}>\mu_{\perp}$ and coherent fragmentation for $q_{\perp}<\mu_{\perp}$. Unlike the factorization scales that we will discuss shortly, $\mu_{\perp}$ is not introduced to renormalize the fragmentation functions but to 
define the perturbative (or non-perturbative) part of the dihadron fragmentation functions. It is quite analogous to the cone-size of jet definitions 10 .

To simplify the discussion in this paper, we will concentrate on the non-singlet fragmentation functions:

$$
D_{N S}^{h_{1}, h_{2}}\left(z_{1}, z_{2}\right)=D_{q}^{h_{1}, h_{2}}\left(z_{1}, z_{2}\right)-D_{\bar{q}}^{h_{1}, h_{2}}\left(z_{1}, z_{2}\right) .
$$

We also use the following convolution notations,

$$
\begin{gathered}
\left.A * B\right|_{a} ^{b}=\int_{a}^{b} \frac{d y}{y^{2}} A\left(z_{1} / y, z_{2} / y\right) B(y) \\
\left.A \mp B\right|_{a} ^{b}=\int_{a}^{b} \frac{d y}{y(1-y)} A\left(z_{1} / y, z_{2} /(1-y)\right) B(y) .
\end{gathered}
$$

The bare fragmentation functions $D_{q, \bar{q}, g}\left(z_{1}, z_{2}\right)$ in Eqs. 4 and 5 are not as yet physical, measurable quantities and are scheme dependent, since the cross sections expressed in terms of them contain collinear divergences. One can however introduce renormalized fragmentation functions such that the double inclusive cross section can be factorized in the form of Eq. 3 and is free of collinear divergences.

Factoring out the $e^{+} e^{-}$annihilation cross section in Eq. 5, we are left with the scale dependent physical fragmentation functions which should be free of collinear divergences. The "non-singlet" physical fragmentation functions are,

$$
\begin{aligned}
& D_{N S}^{h_{1}, h_{2}}\left(z_{1}, z_{2}, Q^{2}\right)=D_{N S}^{h_{1}, h_{2}} \\
& \quad+\left.D_{N S}^{h_{1}, h_{2}} * \frac{\alpha_{s}}{2 \pi} P_{q \rightarrow q g}\right|_{z_{1}+z_{2}} ^{1} \log \left(Q^{2} / m_{g}^{2}\right) \\
& \quad+\left.\left(D_{N S}^{h_{1}} D_{g}^{h_{2}}\right) \approx \frac{\alpha_{s}}{2 \pi} \hat{P}_{q \rightarrow q g}\right|_{z_{1}} ^{1-z_{2}} \log \left(Q^{2} / \mu_{\perp}^{2}\right) \\
& \quad+\left.\left(D_{N S}^{h_{1}} D_{g}^{h_{2}}\right) \approx \frac{\alpha_{s}}{2 \pi} \hat{P}_{q \rightarrow q g}\right|_{z_{1}} ^{1-z_{2}} \log \left(\mu_{\perp}^{2} / m_{g}^{2}\right) \\
& \quad+1 \rightarrow 2
\end{aligned}
$$

Here the switch $1 \rightarrow 2$ is meant solely for the second and third term. Such exchange will be made implicit in the rest of this paper. We will also drop the limits of the convolutions in the notation for brevity. In the case of independent fragmentation, we have also split the expressions into a term that solely includes contributions with $q_{\perp}$ above the scale $\mu_{\perp}$. The second piece contains contributions below $\mu_{\perp}$ and thus receives large corrections from higher order and non-perturbative processes. This piece will have to be absorbed into a redefinition of the bare dihadron fragmentation function.

We now have to introduce the factorization scale $\mu$ and redefine the bare dihadron fragmentation function in terms of a renormalized one and the single fragmentation functions,

$$
\begin{gathered}
D_{N S}^{h_{1}, h_{2}}= \\
\bar{D}_{N S}^{h_{1}, h_{2}}\left(\mu, \mu_{\perp}\right) *\left(1+\frac{\alpha_{s}}{2 \pi} P_{q \rightarrow q g} \log \left(m_{g}^{2} / \mu^{2}\right)+\ldots\right) \\
+D_{N S}^{h_{1}} D_{g}^{h_{2}} \bar{*}\left(\frac{\alpha_{s}}{2 \pi} P_{q \rightarrow q g} \log \left(m_{g}^{2} / \mu_{\perp}^{2}\right)+\ldots\right)
\end{gathered}
$$

Note that the $\log$ in the second term can be separated into two pieces

$$
\log \left(m_{g}^{2} / \mu_{\perp}^{2}\right)=\log \left(m_{g}^{2} / \mu^{2}\right)+\log \left(\mu^{2} / \mu_{\perp}^{2}\right),
$$

with the first one containing the collinear divergence and the second piece defining the independent fragmentation of two collinear partons. In the case where $\mu<\mu_{\perp}$, we essentially have a function that depends on two scales: the collinear divergences that are extracted from the second term in Eq. 8 cannot be factorized out at a scale below $\mu_{\perp}$. However, we may chose to have $\mu>\mu_{\perp}$, in which case the second piece in Eq. 9 is a finite constant that may be simply reabsorbed into the definition of the renormalized fragmentation function. With the factorization scale $\mu$ chosen above the physical scale $\mu_{\perp}$, we may now express Eq. 8 as

$$
\begin{gathered}
D_{N S}^{h_{1}, h_{2}}= \\
\bar{D}_{N S}^{h_{1}, h_{2}}(\mu) *\left(1+\frac{\alpha_{s}}{2 \pi} P_{q \rightarrow q g} \log \left(m_{g}^{2} / \mu^{2}\right)+\ldots\right) \\
+D_{N S}^{h_{1}} D_{g}^{h_{2}} \bar{*}\left(\frac{\alpha_{s}}{2 \pi} P_{q \rightarrow q g} \log \left(m_{g}^{2} / \mu^{2}\right)+\ldots\right) .
\end{gathered}
$$

In what follows we will always compute in the region where $\mu>\mu_{\perp}$.

We may now substitute Eq. 10 into Eq. $\mathbf{7}$ and concentrate on the leading order and leading log (LL) sector of the fragmentation functions (i.e. we only keep terms to order $\left.\alpha_{s}\left(Q^{2}\right) \log \left(Q^{2}\right)\right)$ to get

$$
\begin{aligned}
& D_{N S}^{h_{1}, h_{2}}\left(z_{1}, z_{2}, Q^{2}\right)= \\
& \bar{D}_{N S}^{h_{1}, h_{2}}(\mu) *\left(1+\frac{\alpha_{s}}{2 \pi} P_{q \rightarrow q g} \log \left(Q^{2} / \mu^{2}\right)+\ldots\right) \\
& \quad+D_{N S}^{h_{1}} D_{g}^{h_{2}}\left(\frac{\alpha_{s}}{2 \pi} P_{q \rightarrow q g} \log \left(Q^{2} / \mu^{2}\right)+\ldots\right)
\end{aligned}
$$

The above dihadron fragmentation function in the NLO still contains the bare single fragmentation functions in the contribution from two independent parton fragmentation. 


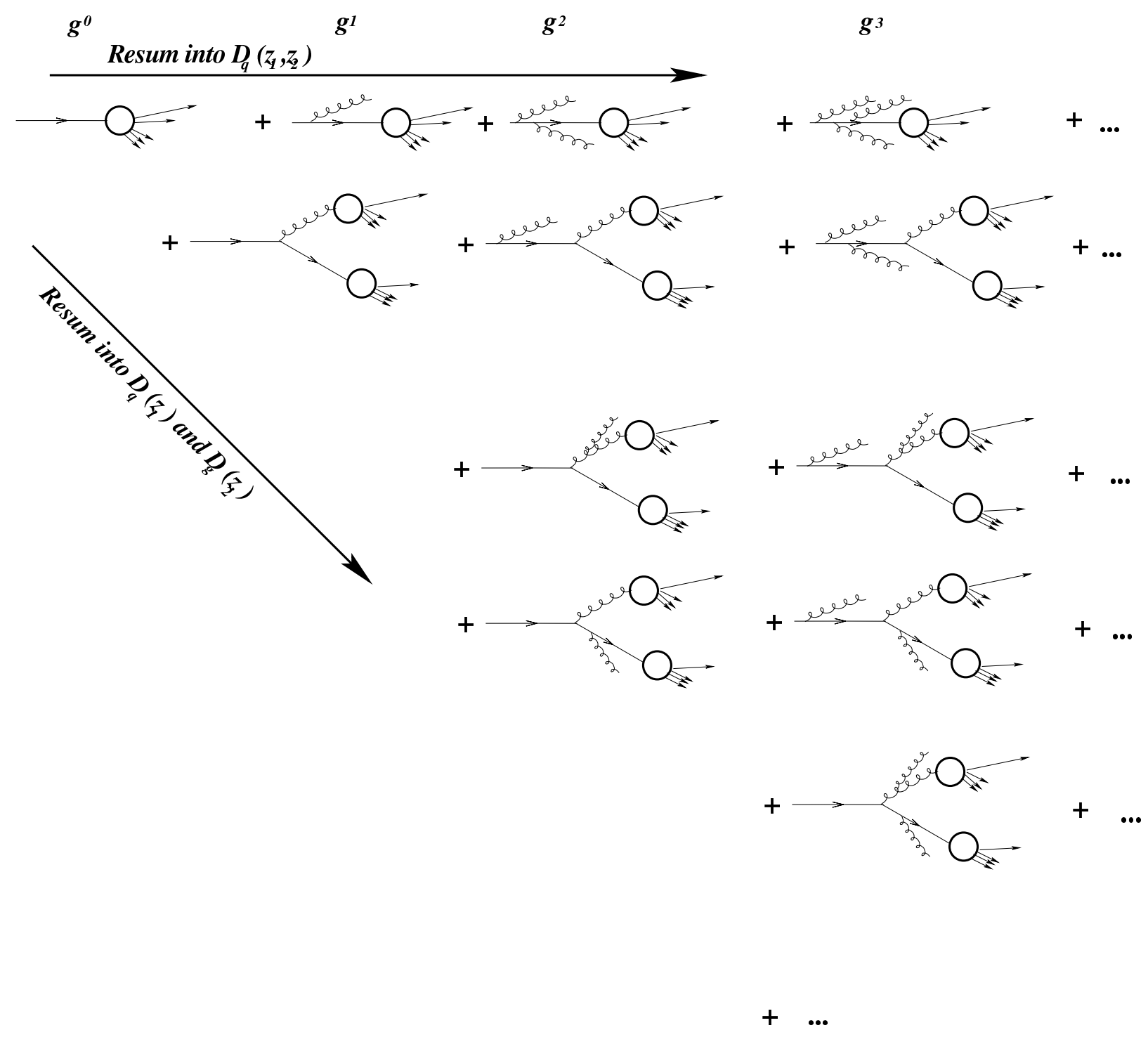

FIG. 1: Diagrams to be resummed iteratively to obtain the evolution of the fragmentation functions.

If we consider higher order processes in which an additional gluon radiation takes place after the split but before independent fragmentation, as shown in Fig. II another collinear divergence will arise. This is exactly the same as in the NLO correction to the single inclusive fragmentation functions. One has to introduce renormalized single hadron fragmentation functions at a factorization scale $\mu_{1}$

$$
D_{N S}^{h_{1}}=\bar{D}_{N S}^{h_{1}}\left(\mu_{1}\right) \otimes\left(1+\frac{\alpha_{s}}{2 \pi} P_{q \rightarrow q g} \log \left(m_{g}^{2} / \mu_{1}^{2}\right)+\ldots\right)
$$

where, the $\otimes$ indicates the regular convolution notation, i.e. $A \otimes B=\int \frac{d y}{y} A(z / y) B(y)$. In addition, the renormalized gluon fragmentation function is defined as,

$$
\begin{aligned}
& D_{g}^{h_{1}}=\bar{D}_{g}^{h_{1}}\left(\mu_{1}^{2}\right) \otimes\left(1+\frac{\alpha_{s}}{2 \pi} P_{g \rightarrow g g} \log \left(m_{g}^{2} / \mu_{1}^{2}\right)+\ldots\right) \\
& \quad+\sum_{q} \bar{D}_{q / \bar{q}}^{h_{1}}\left(\mu_{1}^{2}\right) \otimes \frac{\alpha_{s}}{2 \pi}\left(P_{q \rightarrow q g} \log \left(m_{g}^{2} / \mu_{1}^{2}\right)+\ldots\right),(13)
\end{aligned}
$$

where $\bar{D}_{q / \bar{q}}^{h_{1}}$ represents the quark or antiquark fragmentation function and the sum includes all flavours. The factorization scale $\mu_{1}$ for the single fragmentation functions needs not be the same as the factorization scale for double fragmentation functions.

With both the renormalized single and double hadron fragmentation functions, we obtain the leading log and NLO expressions of the double hadron fragmentation 
functions

$$
\begin{gathered}
D_{N S}^{h_{1}, h_{2}}\left(z_{1}, z_{2}, Q^{2}\right)= \\
\bar{D}_{N S}^{h_{1}, h_{2}}(\mu) *\left(1+\frac{\alpha_{s}}{2 \pi} P_{q \rightarrow q g} \log \left(Q^{2} / \mu^{2}\right)+\ldots\right) \\
+\bar{D}_{N S}^{h_{1}}\left(\mu_{1}\right) \bar{D}_{g}^{h_{2}}\left(\mu_{1}\right) *\left(\frac{\alpha_{s}}{2 \pi} P_{q \rightarrow q g} \log \left(Q^{2} / \mu^{2}\right)+\ldots\right) .
\end{gathered}
$$

In the above discussion we have set $Q^{2}$ to be large such that there exists a hierarchy of scales $\Lambda_{Q C D}^{2}<<\mu_{\perp}^{2}<<$ $Q^{2}$. The above factorization is valid in the regime in which the fragmentation functions are measured at a scale $\mu$ such that $\mu_{\perp}<\mu<<Q$. In this limit we may also set $\mu_{1}=\mu$ to define both single and double fragmentation functions at the same scale. Note that the single fragmentation functions at the new scale $\mu$ differ from those at $\mu_{1}$ at higher order in $\alpha_{s}$ and thus the correction due to these may be ignored in the leading log expressions for large enough $Q^{2}$.

The remaining task in our parton model evaluation of the double hadron fragmentation function is to iterate the radiative process as shown in Fig. II Each of the circular blobs represents a fragmentation function at the scale $\mu$. Differentiating the series with respect to $\log \left(Q^{2}\right)$ followed by a reorganization of the various terms leads to the evolution equation:

$$
\begin{aligned}
\frac{\partial D_{N S}^{h_{1} h_{2}}\left(Q^{2}\right)}{\partial \log Q^{2}} & =\frac{\alpha_{s}}{2 \pi}\left[P_{q \rightarrow q g} * D_{N S}^{h_{1} h_{2}}\left(Q^{2}\right)\right. \\
& \left.+\hat{P}_{q \rightarrow q g} \bar{*} D_{N S}^{h_{1}}\left(Q^{2}\right) D_{g}^{h_{2}}\left(Q^{2}\right)+1 \rightarrow 2\right]
\end{aligned}
$$

Within the framework of the parton model we can picture the process as the free propagation of a parton followed by its fragmentation into hadrons of which two are identified. Fragmentation may be preceded by the radiation of multiple soft gluons (this is the top line in Fig. II. Occasionally the parent parton undergoes a semihard split into two offspring partons which then propagate freely of each other and then fragment independently into hadrons and one hadron from each of these offspring is identified. Prior to their fragmentation, the offspring may radiate multiple soft gluons as well.

The excercise in this section is based on the validity of our assumptions about the nature of the fragmentation process, especially on the validity of Eqs. 35. It was assumed that in progressing from single inclusive to double inclusive cross sections the parton model dynamics would remain the single leading behaviour and more importantly would lead to Eq. 5. Such a proof exists for the single inclusive fragmentation functions that requires an exact operator definition of the single fragmentation functions. One can demonstrate both the factorized form of Eq. II and the evolution equations of the single inclusive fragmentation functions, exactly, as the leading log behaviour at large $Q^{2}$ in an operator formalism (see Refs. \&, C, 20 and Ref. 23). Mounting such a proof for the dihadron fragmentation functions will require us to provide a definition of the dihadron fragmentation function in the operator formalism. This will be the subject of the next section. We will extend the cut-vertex formalism of Mueller 9,20 to dihadron fragmentation in this paper. The factorization of the NLO expressions at leading twist and leading log will be demonstrated in Sec. IV.

\section{THE SINGLE AND DOUBLE FRAGMENTION FUNCTIONS}

In the previous section, we made use of the parton model 7 to motivate a double inclusive fragmentation function and assumed a factorized form as the leading behaviour of the two hadron inclusive cross sections. To prove the factorized behaviour we need to first obtain a consistent definition of the dihadron fragmentation function.

We begin with the matrix element for the electron positron annihilation into a given state of hadrons in the single photon approximation,

$$
\begin{aligned}
& \mathcal{M}_{e+e^{-} \rightarrow S_{h a d}}=e^{2} \int d^{4} y\left\langle S_{h a d}\left|J^{\nu}(y)\right| 0\right\rangle \\
& \times \frac{-i g_{\nu \mu}}{\left(\mathbf{k}_{1}+\mathbf{k}_{2}\right)^{2}} \quad e^{-i\left(\mathbf{k}_{1}+\mathbf{k}_{2}\right) \cdot \mathbf{y}} \bar{v}\left(\mathbf{k}_{2}\right) \gamma^{\mu} u\left(\mathbf{k}_{1}\right) .
\end{aligned}
$$

In the above equation, $J^{\nu}(y)=\sum_{q} \epsilon_{q} \bar{\psi}_{q}(y) \gamma^{\nu} \psi_{q}(y)$ is the hadronic eletromagnetic current and $\mathbf{k}_{1}, \mathbf{k}_{2}$ are the momentum four-vectors of the electron and positron. Here the sum over the number of colors in the fundamental representation of QCD is implied. Squaring the matrix element, summing over all final states of hadrons and averaging over all initial spins of hadrons, one obtains the total cross section for $e^{+} e^{-}$annihilation into hadrons,

$$
\begin{aligned}
\sigma & =\frac{1}{2 s} \sum_{S_{\text {had }}} \int \frac{d^{3} p_{f}}{2 E_{f}(2 \pi)^{3}}(2 \pi)^{4} \delta\left(\mathbf{k}_{1}+\mathbf{k}_{2}-\mathbf{P}_{S_{\text {had }}}\right) \\
& \times \frac{e^{4}}{4\left(\mathbf{q}^{2}\right)^{2}} \mathcal{L}_{\mu \nu}\left\langle 0\left|J^{\mu}(0)\right| S_{\text {had }}\right\rangle\left\langle S_{\text {had }}\left|J^{\nu}(0)\right| 0\right\rangle \\
& =\frac{e^{4}}{2 s \mathbf{q}^{4}} \frac{\mathcal{L}_{\mu \nu} W^{\mu \nu}}{4}
\end{aligned}
$$

where $\mathcal{L}_{\mu \nu}$ is the leptonic tensor and $W^{\mu \nu}$ is the hadronic tensor. The four-momentum of the virtual photon is $\mathbf{q}=$ $\mathbf{k}_{1}+\mathbf{k}_{2} \equiv(Q, 0,0,0)$ and the Mandelstam variable $s=$ $\mathbf{q}^{2}=Q^{2}$. The sum over $S_{\text {had }}$ includes both the sum of the complete set of states and the phase space integration $\prod_{f \in S_{\text {had }}} d^{3} p_{f} / 2 E_{f}(2 \pi)^{3}$.

One can evaluate the single inclusive cross section by summing over all possible hadronic final states that contain the identified hadron $h$. In the leading order and leading twist in a collinear approximation, one can obtain 
Eq. II. In a light-cone gauge $(\mathbf{n} \cdot \mathbf{A}=0)$, the operator expression for the single inclusive fragmentation function at leading twist is obtained as $0,20 \%$

$$
\begin{aligned}
D_{q(\bar{q})}^{h}\left(z_{h}\right) & =\frac{z_{h}^{3}}{2} T_{q(\bar{q})}\left(z_{h}\right) \\
& =\frac{z_{h}^{3}}{4} \int \frac{d^{4} p}{(2 \pi)^{4}} \delta\left(z_{h}-\frac{\mathbf{p}_{h} \cdot \mathbf{n}}{\mathbf{p} \cdot \mathbf{n}}\right) \\
& \times \operatorname{Tr}\left[\frac{\gamma \cdot \mathbf{n}}{\mathbf{p}_{h} \cdot \mathbf{n}} \hat{T}_{q(\bar{q})}\left(\mathbf{p}, \mathbf{p}_{h}\right)\right]
\end{aligned}
$$

where the the Dirac operators $\hat{T}_{q(\bar{q})}\left(\mathbf{p}, \mathbf{p}_{h}\right)$ are given by

$$
\begin{aligned}
\left(\hat{T}_{q}\right)_{\alpha, \beta}\left(\mathbf{p}, \mathbf{p}_{h}\right) & =\int d^{4} x \sum_{S_{h a d}-1}\left\langle 0\left|\psi_{\alpha}(0)\right| \mathbf{p}_{h}, S_{h a d}-1\right\rangle \\
& \times\left\langle\mathbf{p}_{h}, S-1\left|\bar{\psi}_{\beta}(x)\right| 0\right\rangle e^{i \mathbf{p} \cdot \mathbf{x}} \\
\left(\hat{T}_{\bar{q}}\right)_{\alpha, \beta}\left(\mathbf{p}, \mathbf{p}_{h}\right) & =\int d^{4} x \sum_{S_{h a d}-1}\left\langle 0\left|\bar{\psi}_{\beta}(0)\right| \mathbf{p}_{h}, S_{h a d}-1\right\rangle \\
& \times\left\langle\mathbf{p}_{h}, S_{h a d}-1\left|\psi_{\alpha}(x)\right| 0\right\rangle e^{i \mathbf{p} \cdot \mathbf{x}}
\end{aligned}
$$

Here, the sums are taken over all physical final states of hadrons, which always contain, at least, the single hadronic state with momentum $\mathbf{p}_{h}$. In this case $\mathbf{n}$ is chosen such that its spatial components are antiparallel to the spatial components of the observed hadron. This implies $\mathbf{n} \cdot \mathbf{p}_{h}=p_{h}^{+}=\left(p_{h}^{0}+\left|\vec{p}_{h}\right|\right) / 2$. In our choice of light-cone momenta $p^{-}=p^{0}-p_{z}$. The gauge links required to make this expression gauge invariant have been suppressed as they do not contribute to the leading twist fragmentation functions in light-cone gauge.

The fragmentation functions can also be reexpressed in the cut-vertex technique of Mueller. These represent a powerful computational tool that may be used to calculate inclusive cross sections and the scale dependence of these functions in perturbation theory in a diagramatic languange. The Feynman diagrams illustrating the leading order expressions for the single inclusive fragmentation functions are shown in Fig. 2 In this Feynman diagram the rule for the bare quark cut-vertex is

$$
\frac{\gamma \cdot \mathbf{n}}{2 \mathbf{p}_{h} \cdot \mathbf{n}} \delta\left(z_{h}-\frac{\mathbf{p}_{h} \cdot \mathbf{n}}{\mathbf{p} \cdot \mathbf{n}}\right) .
$$

The derivation of operator definitions for dihadron fragmetation functions and the extension of the cut-vertex technique to incorporate these functions is the focus of this section.

We will concentrate on the two-jet events in $e^{+} e^{-}$annihilation and are interested only in two hadron production off one single jet. The fate of the "back-side" jet will not be dwelled over here. We assume that the sum over all hadronic states in $W^{\mu \nu}$ can be simplified into two complete sets of states and that each overlaps independently with the quark and antiquark jet. This assumption neglects the interference between the two jets and is valid in leading log and leading twist. We also assume the duality between the complete hadronic states

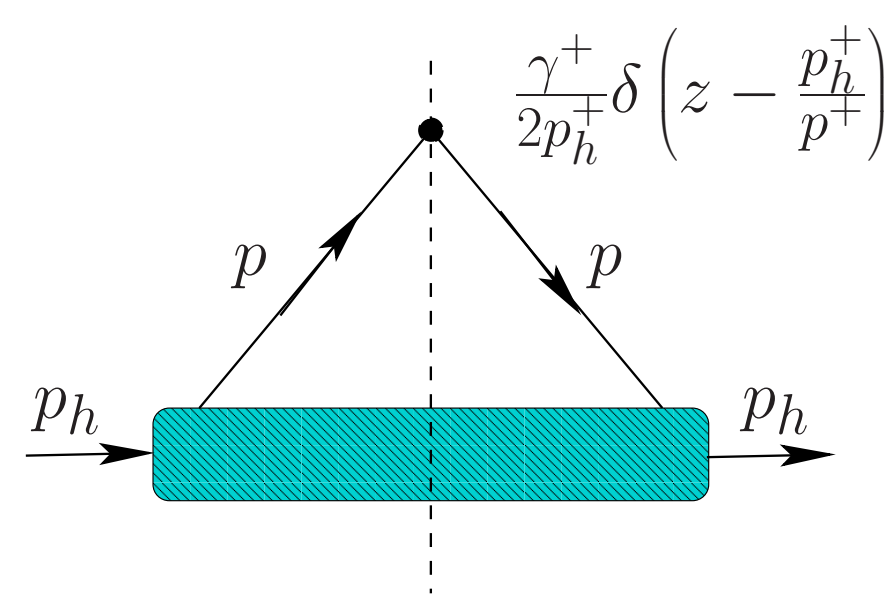

FIG. 2: cut-vertex for quark fragmention function at LO.

and partonic states. Thus the sum over hadronic states in the "back-side" jet will be replaced by partonic states. Under this assumption, we evaluate the hadronic current operator, order by order in $\alpha_{s}$, by expanding the QCD interaction Hamiltonian in the interaction picture. In the leading order (LO), one obtains,

$$
\begin{aligned}
W^{\mu \nu} & =\sum_{S_{\text {had }}-2} \sum_{q} e_{q}^{2} \int \frac{d^{3} p_{1} d^{3} p_{2}}{4 E_{1} E_{2}(2 \pi)^{6}} \\
& \int \frac{d^{3} k}{2 E_{k}(2 \pi)^{3}}(2 \pi)^{4} \delta^{4}\left(\mathbf{q}-\mathbf{p}_{1}-\mathbf{p}_{2}-\mathbf{k}-\sum_{f \in S_{\text {had }}-2} \mathbf{p}_{f}\right) \\
& \times\left\langle 0\left|\bar{\psi}_{q}(0) \gamma^{\mu} \psi_{q}(0)\right| k, p_{1}, p_{2}, S_{\text {had }}-2\right\rangle \\
& \times\left\langle k, p_{1}, p_{2}, S_{\text {had }}-2\left|\bar{\psi}_{q}(0) \gamma^{\nu} \psi_{q}(0)\right| 0\right\rangle
\end{aligned}
$$

In the above equation, $S_{\text {had }}$ is a complete set of hadronic states. In the remaining discussion we will drop the subscript (had). We have extracted two particular hadronic state sums, labeled as $p_{1}, p_{2}$, from the full sum over states $S$. The hadronic tensor may be represented by the Feynman diagram in Fig. $\mathbf{B}$

On Fourier decomposition of one of the quark or antiquark operators, followed by a sum over all spins of the outgoing antiquark (quark) state $\mathbf{k}$, we obtain the 


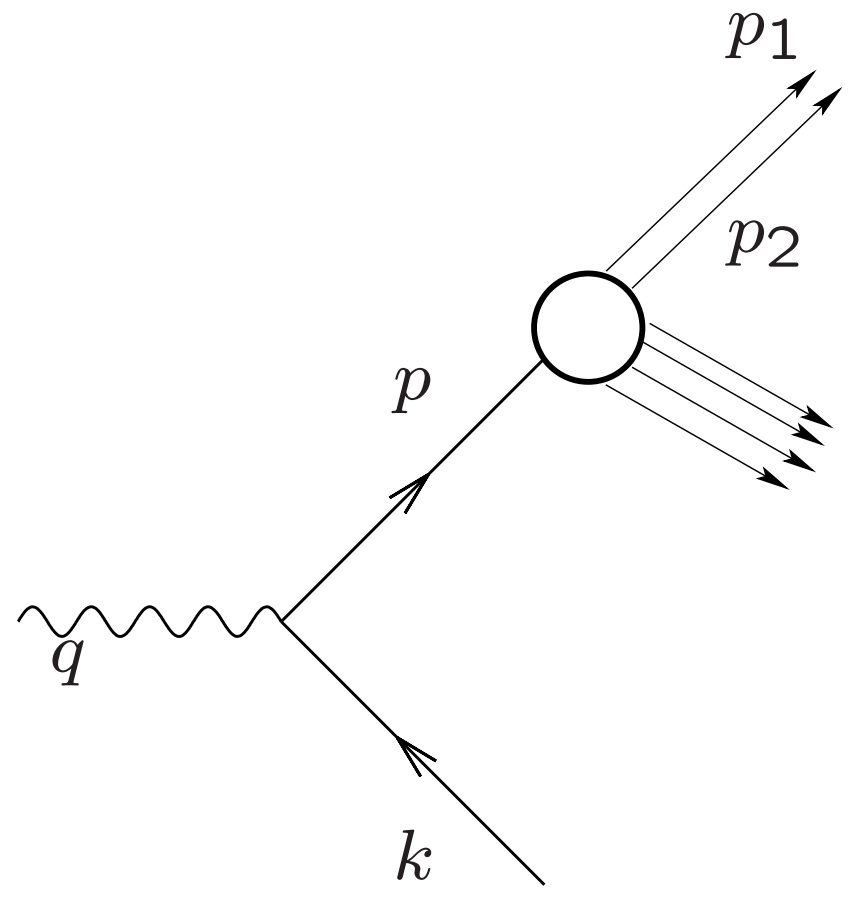

FIG. 3: The leading order Feynman diagram contributing to the double inclusive fragmentation function.

hadronic tensor as,

$$
\begin{aligned}
& W^{\mu \nu}=\sum_{q, S-2} e_{q}^{2} \int \frac{d^{3} p_{1} d^{3} p_{2}}{4 E_{1} E_{2}(2 \pi)^{6}} \\
& \int \frac{d^{4} k}{(2 \pi)^{4}} 2 \pi \delta^{+}\left(\mathbf{k}^{2}\right)(2 \pi)^{4} \delta^{4}\left(\mathbf{q}-\mathbf{p}_{1}-\mathbf{p}_{2}-\mathbf{k}-\mathbf{p}_{S-2}\right) \\
& {\left[\left\{\left\langle 0\left|\bar{\psi}_{q}(0)\right| p_{1}, p_{2}, S-2\right\rangle \gamma^{\mu} \not k \gamma^{\nu}\left\langle p_{1}, p_{2}, S-2\left|\psi_{q}(0)\right| 0\right\rangle\right\}\right.} \\
& \left.+\left\{\gamma^{\mu}\left\langle 0|\psi(0)| p_{1}, p_{2}, S-2\right\rangle\left\langle p_{1}, p_{2}, S-2|\bar{\psi}(0)| 0\right\rangle \gamma^{\nu} \not k\right\}\right] .
\end{aligned}
$$

One may rewrite the $\delta$-function as a four-space integration of an exponent $\exp (-i \mathbf{p} \cdot \mathbf{x})$ that in turn can be used to transform the quark wavefunction operator $\psi(0) \rightarrow \psi(x)$. A shift in the $d^{4} k$ integration can be performed i.e. $\mathbf{k} \equiv \mathbf{q}-\mathbf{p}$. Summing over the spins (and colors) of all the final (parton) states we obtain the general form of the hadronic tensor as

$$
\begin{aligned}
& W^{\mu \nu}=N_{c} \sum_{q} e_{q}^{2} \int \frac{d^{3} p_{1} d^{3} p_{2}}{4 E_{1} E_{2}(2 \pi)^{6}} \int \frac{d^{4} p}{(2 \pi)^{4}} \\
& 2 \pi \delta^{+}\left((\mathbf{q}-\mathbf{p})^{2}\right)\left[\operatorname{Tr}\left\{\hat{T}_{\bar{q}}\left(p ; p_{1}, p_{2}\right) \gamma^{\mu}(\not q-\not p) \gamma^{\nu}\right\}\right. \\
& \left.+\operatorname{Tr}\left\{\hat{T}_{q}\left(p ; p_{1}, p_{2}\right) \gamma^{\nu}(\not q-\not p) \gamma^{\mu}\right\}\right]
\end{aligned}
$$

where the parton-double-hadron overlap matrices are given similarly as for single fragmentation functions as

$$
\begin{aligned}
& {\left[\hat{T}_{\bar{q}}\left(p ; p_{1}, p_{2}\right)\right]^{\alpha \beta}=\int d^{4} x e^{i \mathbf{p} \cdot \mathbf{x}} \sum_{S-2}} \\
& \quad\left\langle 0\left|\bar{\psi}_{q}^{\beta}(x)\right| p_{1} p_{2} S-2\right\rangle\left\langle p_{1} p_{2} S-2\left|\psi_{q}^{\alpha}(0)\right| 0\right\rangle
\end{aligned}
$$

and

$$
\begin{aligned}
& {\left[\hat{T}_{q}\left(p ; p_{1}, p_{2}\right)\right]^{\alpha \beta}=\int d^{4} x e^{i \mathbf{p} \cdot \mathbf{x}} \sum_{S-2}} \\
& \quad\left\langle 0\left|\psi_{q}^{\alpha}(x)\right| p_{1} p_{2} S-2\right\rangle\left\langle p_{1} p_{2} S-2\left|\bar{\psi}_{q}^{\beta}(0)\right| 0\right\rangle,
\end{aligned}
$$

where the two quark field operators have the same color index and average over colors is explicitly implied.

Up to this point the derivation of the factorized double inclusive fragmentation function has followed a path not entirely dissimilar to that of a single fragmentation function. At this point one may introduce the light-cone variables and their ratios,

$$
z_{1}=p_{1}^{+} / p^{+}, z_{2}=p_{2}^{+} / p^{+}
$$

and $z=z_{1}+z_{2}=\left(p_{1}^{+}+p_{2}^{+}\right) / p^{+} \equiv p_{h}^{+} / p^{+}$. Where $p_{h}$ is the total momentum of the pair of hadrons that are identified. We now take the collinear approximation that the hadron momenta $p_{1}, p_{2}, p_{h}$ are almost collinear with respect to the quark (antiquark) momentum at high energies and are thus dominated by their + components for light-cone vector $\mathbf{n}$ chosen in the direction of the outgoing quark or antiquark. Essentially, $p_{1}^{+} \gg p_{1}^{-}, p_{1 \perp}$ and the same is true for $p_{2}$ and $p_{h}$. The overlap matrices $\hat{T}_{\bar{q}}, \hat{T}_{q}$ have a Dirac matrix structure and hence can be decomposed in a basis of products of $\gamma$ matrices $\left(1, \gamma^{\mu}, \sigma^{\mu \nu}, \gamma^{5} \gamma^{\mu}, \gamma^{5}\right)$. The only term of this basis to survive is $\gamma^{\mu}$ : even combinations are set to zero in the trace and combinations containing $\gamma^{5}$ vanish under a spin sum. As $\hat{T}_{\bar{q}}, \hat{T}_{q}$ are scalers and only depend on the three almost collinear momenta, we obtain at leading twist the following decomposition of the overlap matrices:

$$
\begin{aligned}
& \hat{T}_{q}\left(p ; p_{1}, p_{2}\right)=\frac{\not p_{h}}{2} T_{q}\left(p ; p_{1}, p_{2}\right) \text { or } \\
& T_{q}\left(p ; p_{1}, p_{2}\right)=\frac{\operatorname{Tr}\left[\not \hat{T}_{q}\left(p ; p_{1}, p_{2}\right)\right]}{2 \mathbf{n} \cdot \mathbf{p}_{h}}
\end{aligned}
$$

where $T_{q}\left(p ; p_{1}, p_{2}\right)$ is a scalar function. The entire Dirac structure has been extracted into the $\gamma$ matrix.

With the aid of $\delta$-functions, we can introduce the definition of the fractional momenta $z_{1}$ and $z_{2}$ into the 
hadronic tensor:

$$
\begin{aligned}
W^{\mu \nu} & =N_{c} \int_{0}^{1} d z_{1} d z_{2} \theta\left(1-z_{1}-z_{2}\right) \int \frac{d^{3} p_{1} d^{3} p_{2}}{4 E_{1} E_{2}(2 \pi)^{6}}(28) \\
2 & \pi \delta^{+}\left(\mathbf{q}^{2}-2 \mathbf{q} \cdot \frac{\mathbf{p}_{h}}{z}\right) \sum_{q} e_{q}^{2}\left[\operatorname{Tr}\left\{\frac{p_{h}}{2} \gamma^{\mu}\left(\not h-\frac{p_{h}}{z}\right) \gamma^{\nu}\right\}\right. \\
& \times \int \frac{d^{4} p}{(2 \pi)^{4}} T_{\bar{q}}\left(p ;, p_{1}, p_{2}\right) \delta\left(z_{1}-\frac{p_{1}^{+}}{p^{+}}\right) \delta\left(z_{2}-\frac{p_{2}^{+}}{p^{+}}\right) \\
& +\operatorname{Tr}\left\{\frac{\not p_{h}}{2} \gamma^{\nu}\left(\not q-\frac{\not p_{h}}{z}\right) \gamma^{\mu}\right\} \int \frac{d^{4} p}{(2 \pi)^{4}} T_{q}\left(p ;, p_{1}, p_{2}\right) \\
& \left.\times \delta\left(z_{1}-\frac{p_{1}^{+}}{p^{+}}\right) \delta\left(z_{2}-\frac{p_{2}^{+}}{p^{+}}\right)\right] .
\end{aligned}
$$

In collinear approximation, we expand the hard part

$$
\mathcal{H}^{\prime \mu \nu}=\operatorname{Tr}\left[\frac{\not p_{h}}{2} \gamma^{\mu}(\not q-\not p) \gamma^{\nu}\right] \delta^{+}\left((\mathbf{q}-\mathbf{p})^{2}\right)
$$

in the transverse momentum of the hadrons $p_{h \perp}$ and take only the leading term $\mathcal{H}^{\prime \mu \nu}\left(p^{+}\right) \simeq \mathcal{H}^{\prime \mu \nu}\left(p^{+}=p_{h}^{+} / z\right)+\ldots$ This approximation allows us to factor out the hard part from the $d^{4} p$ integral. This is the first step in the factorization of the double hadron inclusive cross section. Based on the collinear approximation we have also dropped the term $\mathbf{p}^{2}$ or $\mathbf{p}_{h}^{2} / z^{2}$ from the argument of the $\delta^{+}$function (i.e. $\mathbf{q}^{2}-2 \mathbf{q} \cdot \mathbf{p}>>\mathbf{p}^{2}$ ). Given the lightcone structure of the four-vector $\mathbf{p}$ a further simplication of the argument of the $\delta$-function may be obtained:

$$
\begin{aligned}
\mathbf{q}^{2}-2 \mathbf{q} \cdot \mathbf{p} & =q^{+} q^{-}-\left(q^{+} p^{-}+q^{-} p^{+}-q_{\perp} \cdot p_{\perp}\right) \\
& =Q^{2}-\left(\frac{Q}{2} p^{-}+Q p^{+}\right), \text {as } q_{\perp}=0 \\
=Q^{2}-Q p^{+} & =Q^{2}-Q \frac{p_{h}^{+}}{z}, \text { as } p^{-}<<p^{+}=p_{h}^{+} / z \\
& \simeq \mathbf{q}^{2}-2 \mathbf{q} \cdot \frac{\mathbf{p}_{h}}{z}
\end{aligned}
$$

With the above simplifications and reorganization of arguments in the two internal $\delta$ functions, the hadronic tensor can be written as

$$
\begin{aligned}
W_{q}^{\mu \nu}= & N_{c} \int_{0}^{1} d z_{1} d z_{2} \theta\left(1-z_{1}-z_{2}\right) \int d \tilde{p}_{1} d \tilde{p}_{2} \\
& 2 \pi \delta^{+}\left(Q^{2}-2 Q \frac{p_{h}^{+}}{z}\right) \mathcal{H}^{\mu \nu} \\
\times & \sum_{q} e_{q}^{2} \int \frac{d^{4} p}{(2 \pi)^{4}}\left[T_{q}\left(p ;, p_{1}, p_{2}\right)+T_{\bar{q}}\left(p ;, p_{1}, p_{2}\right)\right] \\
\times & \frac{p^{+2}}{z_{1} z_{2}} \delta\left(p^{+}-\frac{p_{1}^{+}}{z_{1}}\right) \delta\left(p^{+}-\frac{p_{2}^{+}}{z_{2}}\right),
\end{aligned}
$$

where we have introduced the shorthand notation $d \tilde{p}=$ $d^{3} p /(2 \pi)^{3} 2 E_{p}$. The variable $p^{+}$is overdetermined and thus one of the $\delta$ functions acts really on the integrations external to $d^{4} p$. These may be extracted and further reorganized as follows,

$$
\begin{aligned}
& \ldots \frac{1}{z_{1} z_{2}} \int \frac{d^{4} p}{(2 \pi)^{4}} p^{{ }^{2}} \delta\left(p^{+}-\frac{p_{1}^{+}}{z_{1}}\right) \delta\left(p^{+}-\frac{p_{2}^{+}}{z_{2}}\right) \ldots \\
= & \ldots \frac{1}{z_{1} z_{2}} \delta\left(\frac{p_{1}^{+}}{z_{1}}-\frac{p_{2}^{+}}{z_{2}}\right) \int \frac{d^{4} p}{(2 \pi)^{4}} p^{+2} \delta\left(p^{+}-\frac{p_{1}^{+}}{z_{1}}\right) \ldots \\
= & \ldots \frac{1}{z_{1} z_{2}} \delta\left(\frac{p_{1}^{+}}{z_{1}}-\frac{p_{h}^{+}-p_{1}^{+}}{z_{2}}\right) \int \frac{d^{4} p}{(2 \pi)^{4}} p^{+2} \delta\left(p^{+}-\frac{p_{1}^{+}}{z_{1}}\right) \ldots \\
= & \ldots \frac{1}{z_{1}+z_{2}} \delta\left(p_{1}^{+}-\frac{p_{h}^{+} z_{1}}{z_{1}+z_{2}}\right) \int \frac{d^{4} p}{(2 \pi)^{4}} p^{+2} \delta\left(p^{+}-\frac{p_{1}^{+}}{z_{1}}\right) \ldots \\
= & \ldots \frac{1}{z} \delta\left(p_{1}^{+}-\frac{\left(p_{1}^{+}+p_{2}^{+}\right) z_{1}}{z}\right) \int \frac{d^{4} p}{(2 \pi)^{4}} p^{+2} \delta\left(p^{+}-\frac{p_{h}^{+}}{z}\right) \ldots \\
= & \ldots p_{h}^{+} \delta\left(z_{2} p_{1}^{+}-z_{1} p_{2}^{+}\right) \int \frac{d^{4} p}{(2 \pi)^{4}} \delta\left(z-\frac{p_{h}^{+}}{p^{+}}\right) \ldots,
\end{aligned}
$$

where $z=z_{1}+z_{2}$. Substitution of these expressions into the hadronic tensor $W^{\mu \nu}$ followed by a substitution of $W^{\mu \nu}$ back into Eq. In leads to the following expression for the total cross section for $\epsilon^{+} e^{-}$annihilation,

$$
\begin{aligned}
& \sigma=e^{4} N_{c} \sum_{q} e_{q}^{2} \int d z_{1} d z_{2} \theta\left(1-z_{1}-z_{2}\right) \frac{\mathcal{L}_{\mu \nu} \mathcal{H}^{\mu \nu}(Q)}{8 Q^{6}} \frac{z}{2} \\
& \times \int d \tilde{p}_{1} d \tilde{p}_{2} 2 \pi \frac{z}{2 Q} \delta^{+}\left(p_{h}-z Q / 2\right) p_{h}^{+} \delta\left(z_{2} p_{1}^{+}-z_{1} p_{2}^{+}\right) \\
& \times \int \frac{d^{4} p}{(2 \pi)^{4}} \delta\left(z-\frac{p_{h}^{+}}{p^{+}}\right)\left[T_{q}\left(p ; p_{1}, p_{2}\right)+T_{\bar{q}}\left(p ; p_{1}, p_{2}\right)\right],
\end{aligned}
$$

where the external $\delta$-function has been used to set

$$
\mathcal{H}^{\mu \nu}\left(p_{h}, Q\right) \equiv \frac{z}{2 Q} \delta^{+}\left(p_{h}-z Q / 2\right) \mathcal{H}^{\mu \nu}(Q) \text {. }
$$

The dependence of the hard part on hadronic variables is replaced with the appropriate partonic variables. Differentiating the above equation with respect to $z_{1}, z_{2}$ leads to the double differential cross section outlined in Eq. (3). Before the extraction of the double inclusive fragmentation function, some simplification of the above equation is in order. In contrast to the definition of the single fragmentation function, there are double hadronic integrals $d^{3} p_{1}, d^{3} p_{2}$ and two sets of $\delta$-functions as opposed to one. The cautious reader will note that the overlap matrices $\hat{T}_{q}\left(p ; p_{1}, p_{2}\right), \hat{T}_{\bar{q}}\left(p ;, p_{1}, p_{2}\right)$ [see Eqs. 2526] are dimensionally different from the overlap matrices in the definition of the single inclusive functions (see Ref. 21 ).

To simplify, we begin with a variable transformation. One essentially changes from the set $\left[p_{1 x}, p_{1 y}, p_{1 z}, p_{2 x}, p_{2 y}, p_{2 z}\right]$ to the set $\left[p_{1}, p_{2}, q_{\perp}, \theta_{c m}, \phi_{c m}, \phi_{\perp}\right]$ as illustrated in Fig. 4 This choice is not entirely arbitrary. The discussion of the $\mathrm{NLO}$ in the previous section required us to incorporate transverse momenta $q_{\perp}$ up to a semihard scale $\mu_{\perp}$ into the bare fragmentation function. This particular variable transformation allows us to isolate the $q_{\perp}$ integration.

The new vector $\vec{p}_{h}=\vec{p}_{1}+\vec{p}_{2}$ has the three components of mostly massless four-vectors. The requirement that 


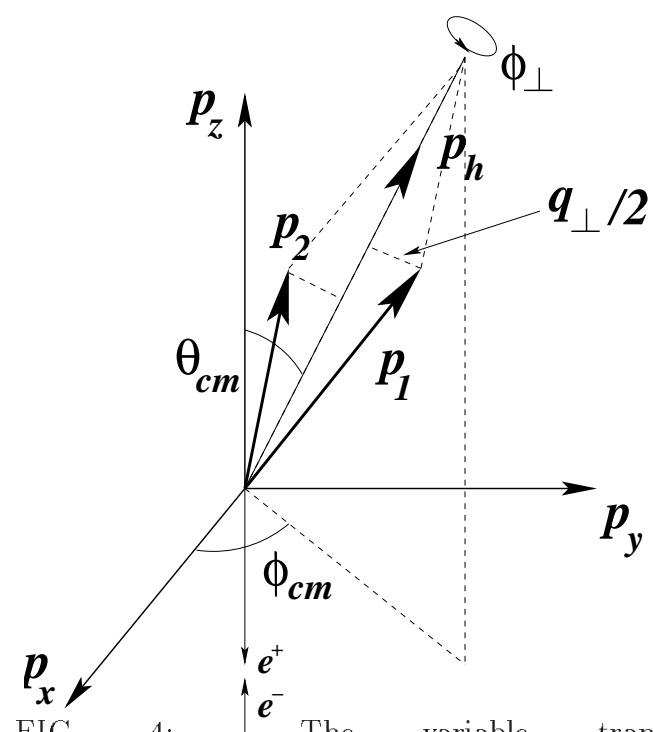

FIG. 4: $\quad \boldsymbol{e}^{-}$The variable transform from $\left[p_{1_{x}}, p_{1_{y}}, p_{1_{z}}, p_{2 x}, p_{2_{y}}, p_{2}\right]$ to $\left[p_{1}, p_{2}, q_{\perp}, \theta_{c m}, \phi_{c m}, \phi_{\perp}\right]$.

$p_{h}^{+}=p_{1}^{+}+p_{2}^{+}$is trivially fulfilled. The new "body-fixed" variable $q_{\perp}$ quantifies the component of $\vec{p}_{1}-\vec{p}_{2}$ that lies on the plane perpendicular to $\vec{p}_{h}$ and $\phi_{\perp}$ is the azimuthal angle of $\vec{q}_{\perp}$ on this plane. The angles $\theta_{c m}$ and $\phi_{c m}$ quantify the direction of $\vec{p}_{h}$ with respect to the $e^{+} e^{-}$beam direction as the $z$ axis. The Jacobian for this tranformation is simply

$$
J=\frac{q_{\perp}}{4}\left(p_{1}+p_{2}\right)^{2},
$$

at leading twist. With these new variables one may easily relate the partonic variable in the hard part with the hadronic variable $\vec{p}_{h}$ as $\vec{p} / z$, i.e. the sum of the 3 momenta of the detected hadrons is collinear with the 3-momenta of the fragmenting quark or antiquark. It may be demonstrated that the corrections to this statement contribute at higher twist. It should be pointed out, in passing, that this is a more accurate statement than the assumption of collinearity between the leading hadron and the fragmenting quark (antiquark) in the case of single fragmentation, as in most cases a dominant part of the jet's momenta is contained in the momenta of the leading hadrons.

With these new variables we can evaluate the inner product of the leptonic tensor and the hard part of the hadronic tensor,

$$
\mathcal{L}_{\mu \nu} \mathcal{H}^{\mu \nu}=4 Q^{4}\left(1+\cos ^{2} \theta_{c m}\right)
$$

and obtain the double differential cross section as

$$
\begin{aligned}
\frac{d \sigma}{d z_{1} d z_{2}}= & \theta\left(1-z_{1}-z_{2}\right) \sum_{q} \frac{e^{4} \epsilon_{q}^{2} N_{c}}{2 s} \int \frac{d p_{1} d p_{2}}{(2 \pi)^{5} 4 p_{1} p_{2}} \\
& d \cos \theta_{c m} d \phi_{c m} d q_{\perp} d \phi_{\perp} \frac{q_{\perp}}{4}\left(p_{1}+p_{2}\right)^{2} \\
\times & \frac{z}{2 Q} \delta^{+}\left(p_{h}-z \frac{Q}{2}\right) \frac{p_{h}^{+}}{z_{1} z_{2}} \delta\left(\frac{p_{1}^{+}}{z_{1}}-\frac{p_{2}^{+}}{z_{2}}\right) \frac{z}{2} \\
\times & \left(1+\cos ^{2} \theta_{c m}\right) \int \frac{d^{4} p}{(2 \pi)^{4}} \delta\left(z-\frac{p_{h}^{+}}{p^{+}}\right) \\
\times & {\left[T_{\bar{q}}\left(p ; p_{1}, p_{2}\right)+T_{q}\left(p ; p_{1}, p_{2}\right)\right] . }
\end{aligned}
$$

Assuming that the overlap matrices, $T_{\bar{q}}\left(p ; p_{1}, p_{2}\right)$ and $T_{q}\left(p ; p_{1}, p_{2}\right)$, are independent of the angles, $\theta_{c m}, \phi_{c m}$ and $\phi_{\perp}$, one can carry out the integrations over these variables. It may once again be stipulated that the following factorization is being performed and expected to succeed only at high energies and momenta (large $Q^{2}$ limit). In this limit we note

$$
p^{+} \simeq p\left[1-O\left(\frac{q_{\perp}^{2}}{p^{2}}\right)\right] .
$$

Hence, in the collinear limit $p, p^{+}>>q_{\perp}$, one may substitute $p \rightarrow p^{+}$in the entire integrand. Regardless of the presence of $\delta$ functions it may be demonstrated that the correction to this approximation is suppressed by at least a power of $Q^{2}$. The two remaining $\delta$-functions external to the $d^{4} p$ integration, i.e.

$$
\delta^{+}\left(p_{1}+p_{2}-z \frac{Q}{2}\right) \delta\left(\frac{p_{1}^{+}}{z_{1}}-\frac{p_{2}^{+}}{z_{2}}\right),
$$

may be used to evaluate the $p_{1}$ and $p_{2}$ integrals. Factoring out the LO total $e^{+} e^{-}$annihilation cross section $\sigma_{0}^{q \bar{q}}$, we obtain the factorized double differential cross section in a form similar to the structure of Eq. 3 as,

$$
\begin{aligned}
\frac{d \sigma}{d z_{1} d z_{2}} & =\sum_{q} \sigma_{0}^{q \bar{q}} \int \frac{q_{\perp} d q \perp}{4(2 \pi)^{2}} \frac{z^{4}}{4 z_{1} z_{2}} \int \frac{d^{4} p}{(2 \pi)^{4}} \\
& \times\left[T_{\bar{q}}\left(p ; p_{1}, p_{2}\right)+T_{q}\left(p ; p_{1}, p_{2}\right)\right] \delta\left(z-\frac{p_{h}^{+}}{p^{+}}\right) .
\end{aligned}
$$

We thus arrive at the definition of the leading order double inclusive fragmentation function as

$$
\begin{aligned}
D_{q}^{h_{1}, h_{2}}\left(z_{1}, z_{2}\right) & =\int \frac{d q_{\perp}^{2}}{8(2 \pi)^{2}} \frac{z^{4}}{4 z_{1} z_{2}} \int \frac{d^{4} p}{(2 \pi)^{4}} \\
& \times T_{q}\left(p ; z_{1} p, z_{2} p\right) \delta\left(z-\frac{p_{h}^{+}}{p^{+}}\right) .
\end{aligned}
$$

In cut-vertex notation, the dihadron fragmentation function may also be expressed by the following equation

$$
D_{q}^{h_{1} h_{2}}\left(z_{1}, z_{2}\right)=\frac{z^{4}}{4 z_{1} z_{2}} \tilde{T}_{q}\left(z_{1}, z_{2}\right)
$$


where $\tilde{T}\left(z_{1}, z_{2}\right)$ is given by the diagram in Fig. 5 Note that the bare cut-vertex has undergone no change as compared to the single hadron fragmentation function, except it takes as input the sum of the fractional momenta $z=z_{1}+z_{2}$. The soft hadronic sector is slightly modified by the exclusion of two hadronic momenta (instead of one) and the integration of the transverse momenta $q_{\perp}$.

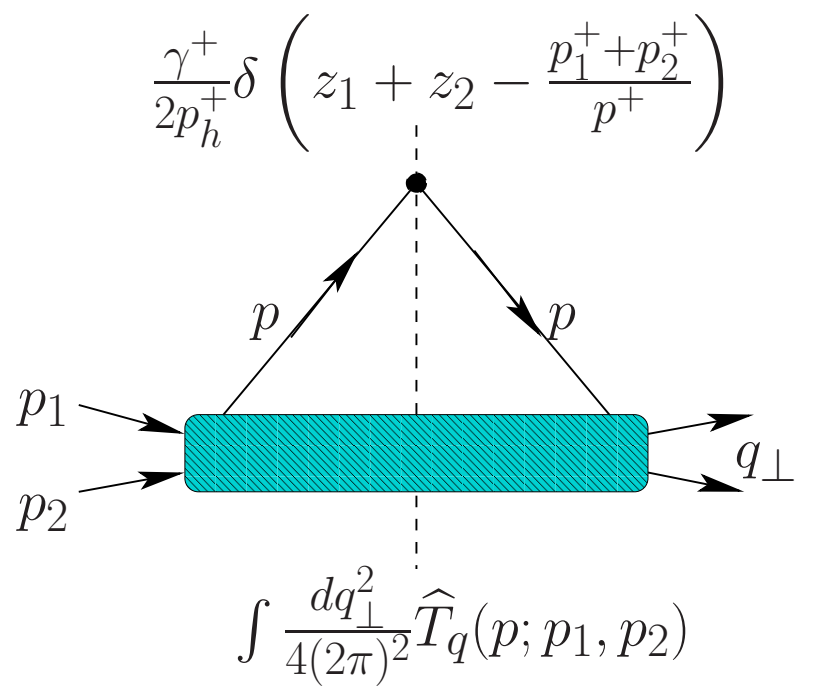

FIG. 5: The cut-vertex representation of the dihadron fragmentation function.

Note that the definition of the dihadron fragmentation functions in their factorized form as in Eq. B seems to depend on our choice of variable transformation (see. Fig. 4. It may indeed be possible to use a different variable transformation and obtain a similar factorization. The sole constraint on the choice of transformation to be used is based on the factorization of the NLO expressions into a form similar to that of Eq. 5, with the same definition of the double fragmentation functions. We will demonstrate this in the next section. A second motivation for this choice of variables is the ability to isolate the transverse momentum $q_{\perp}$, which is integrated over. In the operator definition of the dihadron fragmentation functions, $q_{\perp}$ is generated non-perturbatively. So we will call it intrinsic. The upper limit of integration of this intrinsic $q_{\perp}$ has not been specified in Eq. 37. From our discussion in the previous section of the parton model and of the NLO processes in the next section, the upper limit may be set as $\mu_{\perp}$. We will assume that hadron pairs with the relative transverse momentum $q_{\perp}>\mu_{\perp}$ are generated only perturbatively.

\section{CROSS SECTION AT NLO AND DGLAP EVOLUTION}

With the definition of the dihadron fragmentation functions in the operator formalism, which is shown to factorize from the hard parton cross section in the LO, we are ready to study the DGLAP evolution of the dihadron fragmentation functions by computing the double inclusive cross section at next to leading order (NLO). The calculation will also justify the factorized form of Eq. 5 and our LO definition of the dihadron fragmentation functions.

The NLO matrix element of $e^{+} e^{-}$annihilation process can be obtained from the perturbative expansion of the $S$-matrix with an interaction Hamiltonian which includes an interaction potential corresponding to a quark of color $j$ interacting with an antiquark of color $i$ (or visa versa) and a gluon of color $a$ :

$$
\mathcal{H}_{s}=i g t_{i, j}^{a} \bar{\psi}_{i} \gamma^{\rho} \psi_{j} A_{\rho}^{a}
$$

The $t_{i, j}^{a}$ 's represent the Gell-Mann matrices.

The DGLAP evolution arises from collinear gluon bremstrahlung in the final state of two jet events. As in the case at LO in the previous section, we can again neglect the interference between the two jets in either the partonic or the hadronic level in the leading log and leading twist approximation. Therefore, the sum over all hadronic states is decomposed into the sum over two complete sets of hadronic states, each overlapping with one of the opposite-moving jets. Invoking the partonhadron duality, the sum over the hadronic states in the "back-side" jet can be replaced by a sum over partonic states. Therefore, partonic processes within the "backside" jet will not contribute to the evolution of the fragmentation function in the opposite side which has been defined within a rather strict collinear approximation. As in the case of single fragmentation function, we will also assume, in addition, that there is no interference between the fragmentation of the leading parton and the radiated gluon that has a minimum transverse momentum set by the factorization scale $\mu$.

Hence, in NLO, the sum over all states may be expressed as

$$
|S\rangle=\left|k_{q(\bar{q})}\right\rangle \times\left|S-2, \mathbf{p}_{1} \mathbf{p}_{2}\right\rangle
$$

Depending on how different operators are contracted with the outgoing hadronic state $|S-2\rangle$, we may identify three different cases:

$$
\begin{aligned}
\left|S-2, \mathbf{p}_{1} \mathbf{p}_{2}\right\rangle & =\left|S-2, \mathbf{p}_{1} \mathbf{p}_{2}\right\rangle \times\left|(p-l)_{g}\right\rangle \\
& +\left|S-2, \mathbf{p}_{1} \mathbf{p}_{2}\right\rangle \times\left|(p-l)_{\bar{q}(q)}\right\rangle \\
& +\left|S-1, \mathbf{p}_{1}\right\rangle \times\left|S-1, \mathbf{p}_{2}\right\rangle .
\end{aligned}
$$

These cases differ in the partonic operator that contracts with the hadronic state. In the above, the parton with momentum $\mathbf{k}$ which proceeds in a direction opposite to that of the identified hadrons alternates between a quark 
and an antiquark. In the rest of this paper, we will always take it as an antiquark in order to focus on the quark fragmentation function. The case for the antiquark fragmentation function will be formally identical to that of the quark.

In the following subsections, we will evaluate contributions from these different cases in detail. Roughly speaking, the first line of Eq. 40 represents contributions where the fragmenting quark undergoes a split into a quark and a gluon and the two identified hadrons emanate from the quark offspring. The second contribution represents the case where both identified hadrons emanate from the gluon. The last contribution represents the case where one hadron emanates from each of the quark and gluon offspring.

\section{A. NLO contribution from quark fragmentation}

Proceeding with the evaluation of the double differential cross section at next-to-leading order, the focus in this subsection will be on isolating contributions to the cross section that contain an explicit expression for a dihadron fragmentation function of a quark. The outstate in this subsection will solely be restricted to the first line of Eq. 40]. The instate is simply that of an incoming $\epsilon^{+} e^{-}$pair. Insertion of the interaction operator density $T\left[\mathcal{H}_{e^{+} e^{-} \gamma} \mathcal{H}_{q \bar{q} \gamma} \mathcal{H}_{q \bar{q} g}\right]$, followed by a contraction of the outgoing antiquark operator with the states $\left|k_{\bar{q}}\right\rangle$ and gluon operator with $\left|(p-l)_{g}\right\rangle$, leads to the following matrix element:

$$
\begin{aligned}
\mathcal{M}^{i} & =i \sum_{q} e_{q} e^{2} g t^{a} \bar{v}_{k_{2}} \gamma^{\mu} u_{k_{1}} \frac{g_{\mu \nu}}{\mathbf{q}^{2}+i \epsilon} \\
& \times\left\langle\mathbf{p}_{1} \mathbf{p}_{2} S-2\left|\bar{\psi}_{q}(0)\right| 0\right\rangle\left\{\frac{\gamma^{\nu}(-\not \mathbf{q}+\boldsymbol{\eta}) \boldsymbol{\gamma}^{\rho}}{(\mathbf{q}-\mathbf{l})^{2}+i \epsilon}\right. \\
& \left.+\frac{\gamma^{\rho}(\not \mathbf{q}-\mathbf{k}) \gamma^{\nu}}{(\mathbf{q}-\mathbf{k})^{2}+i \epsilon}\right\} v^{s}(k) \varepsilon_{a \rho}^{\lambda^{*}}(p-l) \\
& \times(2 \pi)^{4} \delta^{4}(\mathbf{q}-\mathbf{k}-\mathbf{p}-\mathbf{l}) .
\end{aligned}
$$

In the above equation there are two terms with differernt momentum dependences within the curly brackets. The reader will readily note that the second term is the Feynman rule for the process indicated in the upper panel of Fig. 6 while the other term consists of the Feynman diagram where the gluon is radiated from the antiquark line, as shown in the lower panel of Fig. 6

In computing the NLO cross section, one may once again factorize the cross section into a leptonic and a hadronic piece [see Eq. [7] ]. Summing over all final states of the outgoing antiquark, gluon and hadrons from the fragmenting quark (besides $h_{1}$ and $h_{2}$ ) followed by an incorporation of minor simplifications, the hadronic
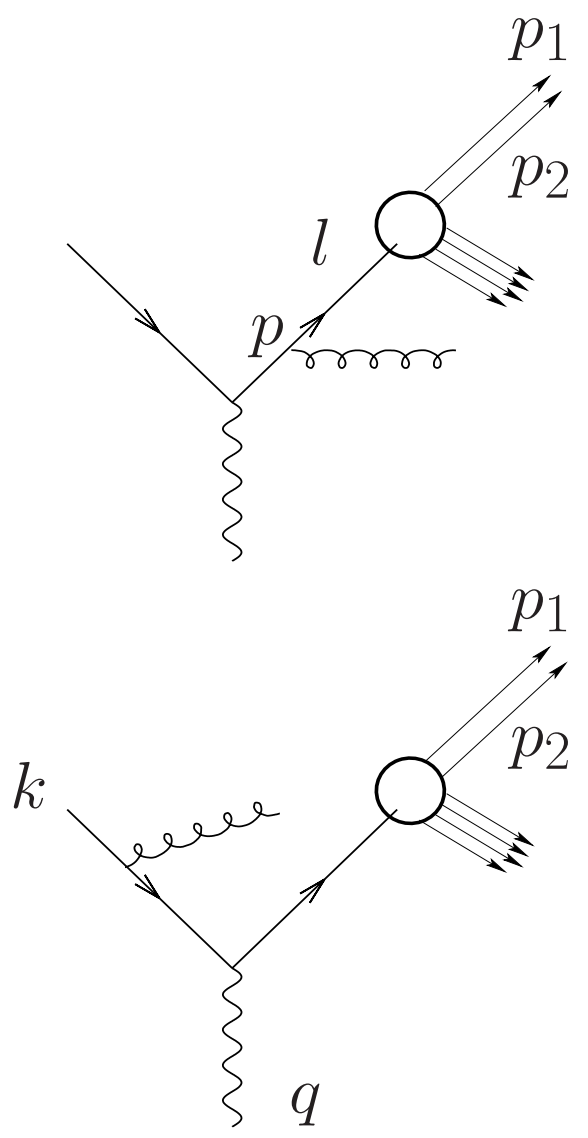

FIG. 6: The leading log contribution to the NLO modification of the quark fragmentation function.

tensor may be expressed as

$$
\begin{aligned}
W^{\alpha \nu} & =\int \frac{d^{3} p_{1} d^{3} p_{2}}{(2 \pi)^{6} 4 E_{1} E_{2}} \int \frac{d^{4} l}{(2 \pi)^{4}} \int \frac{d^{4} p}{(2 \pi)^{4}} \\
& \times 2 \pi \delta^{+}\left((\mathbf{p}-\mathbf{l})^{2}\right) 2 \pi \delta^{+}\left((\mathbf{q}-\mathbf{p})^{2}\right) g^{2} N_{c} C_{F} d_{\rho \sigma}(p-l) \\
& \times \operatorname{Tr}\left[(\boldsymbol{q}-\not{p})\left\{\frac{\gamma^{\alpha} \not \mathbf{p} \gamma^{\rho}}{\mathbf{p}^{2}-i \epsilon}+\frac{\gamma^{\rho}(\mathbf{p}-\not \mathbf{q}) \gamma^{\alpha}}{(\mathbf{l}-\mathbf{q})^{2}-i \epsilon}\right\}\right. \\
& \left.\times \hat{T}_{q}\left(\mathbf{l} ; \mathbf{p}_{1}, \mathbf{p}_{2}\right)\left\{\frac{\gamma^{\sigma} \not \mathbf{p} \gamma^{\nu}}{\mathbf{p}^{2}+i \epsilon}+\frac{\gamma^{\nu}(\mathbf{p}-\not{q}) \gamma^{\sigma}}{(\mathbf{l}-\mathbf{q})^{2}+i \epsilon}\right\}\right] .
\end{aligned}
$$

In the above equation the color factor $N_{c} C_{F}$ comes from the factor $\operatorname{Tr}\left[t^{a} t^{b}\right] \delta^{a b}$. For brevity, we have omitted in the above the sum over quark flavors weighted with fractional charge, $\sum_{q} e_{q}^{2}$. The sum over polarizations of the gluon leads to the factor $d_{\rho \sigma}$. In light-cone gauge $d_{\rho \sigma}$ is given as

$$
d_{\rho \sigma}(p-l)=g_{\rho \sigma}-\frac{(p-l)_{\rho} n_{\sigma}+(p-l)_{\sigma} n_{\rho}}{(\mathbf{p}-\mathbf{l}) \cdot \mathbf{n}}
$$

The overlap matrix element $\hat{T}_{q}\left(\mathbf{l} ; \mathbf{p}_{1}, \mathbf{p}_{2}\right)$ is the same as defined in the previous section, with the final fragmenting 
quark momentum reduced to 1 . The remaining portion of Eq. 42 is also easy to trace. The two $\delta$ functions essentially stipulate that the gluon and antiquark be released onshell. The first set of terms in the curly brackets represents the process indicated by upper panel of Fig. 6 The second set of terms indicates the case where the gluon is emitted from the outgoing antiquark, with all other features remaining unchanged, e.g., the fragmenting quark has momentum $\mathbf{l}$, the gluon still has a momentum of $\mathbf{p}-\mathbf{l}$.

Within the collinear approximation, we again assume that $l^{+}, p_{h}^{+} \gg>l^{-}, p_{h}^{-}, l_{\perp}, q_{\perp}$. As a result, the overlap matrix element may be factorized via the following approximation:

$$
\begin{aligned}
\hat{T}_{q}\left(\mathbf{l} ; \mathbf{p}_{1}, \mathbf{p}_{2}\right) & =\int d^{4} x e^{i \mathbf{1} \cdot \mathbf{x}} \sum_{S-2} \\
\times\left\langle 0\left|\psi_{q}(x)\right| \mathbf{p}_{1}, \mathbf{p}_{2}, S-2\right\rangle\left\langle\mathbf{p}_{1}, \mathbf{p}_{2}, S-2\left|\psi_{q}(0)\right| 0\right\rangle & \\
& \simeq \frac{\mathbf{p}_{h}}{2} T_{q}\left(\mathbf{l}, \mathbf{p}_{1}, \mathbf{p}_{2}\right)
\end{aligned}
$$

Within the collinear approximation, we assume that the momentum of the final fragmenting quark is collinear with that of the hadrons emerging from the parton fragmentation. We thus define a new fractional momentum, $z^{\prime}=p_{h}^{+} / l^{+}$. This allows the partonic four-momentum vector 1 to be replaced with the hadronic four-vector: i.e. $\mathbf{l}=\mathbf{p}_{h} / z^{\prime}$.

As a result the factor $(\mathbf{l}-\mathbf{q})^{2}$ is approximated as:

$$
(\mathbf{l}-\mathbf{q})^{2} \simeq Q^{2}-2 Q l^{+} \simeq Q^{2}-2 Q p_{h}^{+} / z^{\prime}
$$

Unlike the case of the LO process, the transverse momentum of the quark which emanates from the electromagnetic vertex is non-vanishing, $p_{\perp} \neq 0$ (this is also the transverse momentum carried by the gluon). As a result, the negative longitudinal momentum $p^{-}$is constrained by one of the $\delta^{+}$functions as,

$$
\begin{aligned}
\left(\mathbf{p}-\mathbf{p}_{h} / z^{\prime}\right)^{2} & \simeq 2 p^{+} p^{-}-p_{\perp}^{2}-2 p^{-} p_{h}^{+} / z^{\prime}=0 \\
& \Rightarrow p^{-}=\frac{p_{\perp}^{2}}{2\left(p^{+}-p_{h}^{+} / z^{\prime}\right)}
\end{aligned}
$$

The hadron fractional forward light-cone momentum is still defined as $z=p_{h}^{+} / p^{+}$as in the LO case. At leading twist one may replace all occurences of $\mathbf{p}_{h}$ with $z^{\prime} 1$. The corrections to this approximation are down by powers of $Q^{2}$. Incorporating the above approximations into the expression for the hadronic tensor, we obtain:

$$
\begin{aligned}
& W^{\alpha \nu}=\int d z_{1} d z_{2} d z^{\prime} \int \frac{d^{3} p_{1} d^{3} p_{2}}{(2 \pi)^{6} 4 E_{1} E_{2}} \int \frac{d^{4} p}{(2 \pi)^{4}} \\
& \times g^{2} N_{c} C_{F} \delta\left(z_{1}-p_{1}^{+} / p^{+}\right) \delta\left(z_{2}-p_{2}^{+} / p^{+}\right) d_{\rho \sigma}(p-l) \\
& \times 2 \pi \delta^{+}\left(2 p^{-}\left(p^{+}-l^{+}\right)-p_{\perp}^{2}\right) 2 \pi \delta^{+}\left(Q^{2}-2 Q p^{+}\right) \\
& \times \operatorname{Tr}\left[(\not \mathbf{q}-\not \mathbf{p})\left\{\frac{\gamma^{\alpha} \not \mathbf{p} \gamma^{\rho}}{p_{\perp}^{2} l^{+} /\left(p^{+}-l^{+}\right)}+\frac{\gamma^{\rho}(\boldsymbol{\gamma}-\not \mathbf{q}) \gamma^{\alpha}}{Q^{2}-2 Q l^{+}}\right\}\right. \\
& \times\left.\operatorname{y}_{\frac{z^{\prime}}{2}}\left\{\frac{\gamma^{\sigma} \not \boldsymbol{p}^{\nu}}{p_{\perp}^{2} l^{+} /\left(p^{+}-l^{+}\right)}+\frac{\gamma^{\nu}(\boldsymbol{\gamma}-\not \mathbf{q}) \gamma^{\sigma}}{Q^{2}-2 Q l^{+}}\right\}\right]_{\mathbf{l}=\mathbf{p}_{h} / z^{\prime}} \\
& \times \int \frac{d^{4} l}{(2 \pi)^{4}} \delta\left(z^{\prime}-\frac{p_{h}^{+}}{l^{+}}\right) T_{q}\left(\mathbf{l} ; \mathbf{p}_{1}, \mathbf{p}_{2}\right) .
\end{aligned}
$$

A careful study of Eq. 47 reveals that the leading log contributions are dominated by the region where $p_{\perp} \rightarrow 0$. As a result, the part of $W^{\alpha \nu}$ which represents the square of the process in the lower panel of Fig. 6 has no leading log contribution. The leading log contribution comes from the square of the first term corresponding to the square of the process in the upper panel of Fig. 6 In the light-cone gauge, the interference terms have no contribution at leading log as in the case of single fragmentation functions (see chapter 3 of Ref. 18). This can be demonstrated to hold at leading twist merely by completing the trace as indicated in Eq. 47 and extracting the $p_{\perp}^{2}$ dependence from the numerators.

The remaining factorization into the hard and soft piece proceeds as in the $\mathrm{LO}$ case, leading to the hadronic tensor at NLO at leading log and leading twist,

$$
\begin{aligned}
W^{\alpha \nu} & =\int d z_{1} d z_{2} d z^{\prime} \int \frac{d^{3} p_{1} d^{3} p_{2}}{(2 \pi)^{6} 4 E_{1} E_{2}} \int \frac{d^{4} p}{(2 \pi)^{4}} \\
& \times g^{2} N_{c} C_{F} \delta\left(z_{1}-p_{1}^{+} / p^{+}\right) \delta\left(z_{2}-p_{2}^{+} / p^{+}\right) \\
& \times \frac{2 \pi \delta^{+}\left(p^{-}-\frac{p_{\perp}^{2}}{2\left(p^{+}-l^{+}\right)}\right)}{2\left(p^{+}-l^{+}\right)} 2 \pi \delta^{+}\left(Q^{2}-\frac{2 Q p_{h}^{+}}{z}\right) \\
& \times \operatorname{Tr}\left[d_{\rho \sigma}(p-l)(\not \mathbf{q}-\not \mathbf{p}) \frac{\gamma^{\alpha} \not \mathbf{p} \gamma^{\rho} \not\left(z^{\prime} / 2\right) \gamma^{\sigma} \not \mathbf{p} \gamma^{\nu}}{\left[p_{\perp}^{2} l^{+} /\left(p^{+}-l^{+}\right)\right]^{2}}\right]_{\mathbf{l}=\mathbf{p}_{h} / z^{\prime}} \\
& \times \int \frac{d^{4} l}{(2 \pi)^{4}} \delta\left(z^{\prime}-\frac{p_{h}^{+}}{l^{+}}\right) T_{q}\left(\mathbf{l} ; \mathbf{p}_{1}, \mathbf{p}_{2}\right)
\end{aligned}
$$

The trace of the Dirac matrices can be carried out by brute force. The leading-twist part can be obtained in a more straightforward way by rewriting the matrices within the trace symbolically as

$$
(\not-\not p) \gamma^{\alpha} \hat{C} \gamma^{\nu}
$$

The leading twist portion of the matrix $\hat{C}$, may be written as

$$
\hat{C}=\frac{p_{h}}{2} C
$$


with the factor $C$ expressed as a trace:

$$
C=\operatorname{Tr}\left[d_{\rho \sigma} \frac{\gamma^{+}}{2 p_{h}^{+}} \frac{\not \mathbf{p}}{\mathbf{p}^{2}} \gamma^{\rho} \gamma \frac{z^{\prime}}{2} \gamma^{\sigma} \frac{\not \mathbf{p}}{\mathbf{p}^{2}}\right]_{\mathbf{l}=\mathbf{p}_{h} / z^{\prime}},
$$

where $\mathbf{p}^{2}=p_{\perp}^{2} l^{+} /\left(p^{+}-l^{+}\right)$. This procedure is very similar to the one used to construct the collinear approximation to the overlap matrices $\hat{T}_{q}\left(p ; p_{1}, p_{2}\right)$. One can complete the above trace and obtain the regular splitting function $\left(1+y^{2}\right) /(1-y)$, where $y=z^{\prime} / z$. It represents the probability for the radiation of a gluon from a quark prior to its fragmentation.

Using the above approximation one may extract the same hard part as in Eq. 29],

$$
\begin{aligned}
\mathcal{H}^{\prime \alpha \nu} & =\operatorname{Tr}\left[(\boldsymbol{q}-\not \mathbf{p}) \gamma^{\alpha} \frac{p_{h}^{\nu}}{2} \gamma^{\nu}\right] \delta\left(\left(\mathbf{q}-\mathbf{p}_{h} / z\right)^{2}\right) \\
& =\mathcal{H}^{\alpha \nu} \delta\left(\left(\mathbf{q}-\mathbf{p}_{h} / z\right)^{2}\right)
\end{aligned}
$$

Extracting this hard part and comparing with Eqs. 28 31 and the resulting cut-vertex diagram in Fig. 5 we note that the soft part of $\mathrm{Eq}$. 48 begins to display a structure as illustrated in Fig. $\mathbf{7}$

To complete the calculation and obtain a factorized form of the NLO contribution to the double inclusive cross section, the integration over the tranverse momentum of the identified hadrons will have to be factored into the fragmentation function. Similarly as in the case of LO calculation, one has to transform the basis of the momentum integrations of the two identified hadrons to the basis indicated in Fig. 4

In the steps leading to Eq. 48 , the approximation that the momenta of the hadrons and the fragmenting parton are collinear has been made. Particular among these are the approximations that $l^{+}>>l_{\perp}, l^{-}$. These essentially indicate that the invariant mass of the final fragmenting quark is negligible compared to its forward light-cone momentum. This is identical to the approximation made on the momentum $\mathbf{p}$ in the LO calculation (see Fig. 3. Since the NLO process has a collinear divergence when $p_{\perp}^{2} \rightarrow 0$, we will only consider the leading $\log$ contribution.

We thus obtain the following factorized form for the hadronic tensor:

$$
\begin{aligned}
W^{\alpha \nu} & =\int d z_{1} d z_{2} \int \frac{d p_{1} d p_{2}}{(2 \pi)^{4} 4 p_{1} p_{2}} d \cos \theta_{c m} d \phi_{c m} d \phi_{\perp} \\
& \times g^{2} N_{c} C_{F} \int_{z}^{1} \frac{d y}{y^{2}} z \int \frac{d p^{+} d p^{-} d p_{\perp}^{2}}{(2 \pi)^{4}} 2 \pi \mathcal{H}^{\alpha \nu} \delta\left(\left(\mathbf{p}-\mathbf{l}^{2}\right)\right. \\
& \times C p^{+2} \delta\left(p_{1}^{+}-z_{1} p^{+}\right) \delta\left(p_{2}^{+}-z_{2} p^{+}\right) \frac{z}{2 y} \\
& \times \int \frac{d q_{\perp}^{2}}{4(2 \pi)^{2}} \int \frac{d^{4} l}{(2 \pi)^{4}} \delta\left(z^{\prime}-\frac{p_{h}^{+}}{l^{+}}\right) T_{q}\left(\mathbf{l} ; \mathbf{p}_{1}, \mathbf{p}_{2}\right)
\end{aligned}
$$

Further factorization of $\mathcal{H}^{\alpha \nu}$ from the $d^{4} p$ integration leads to the factorized form for the NLO correction to the dihadron fragmentation function as illustrated in Fig. $\mathbf{n}$ in

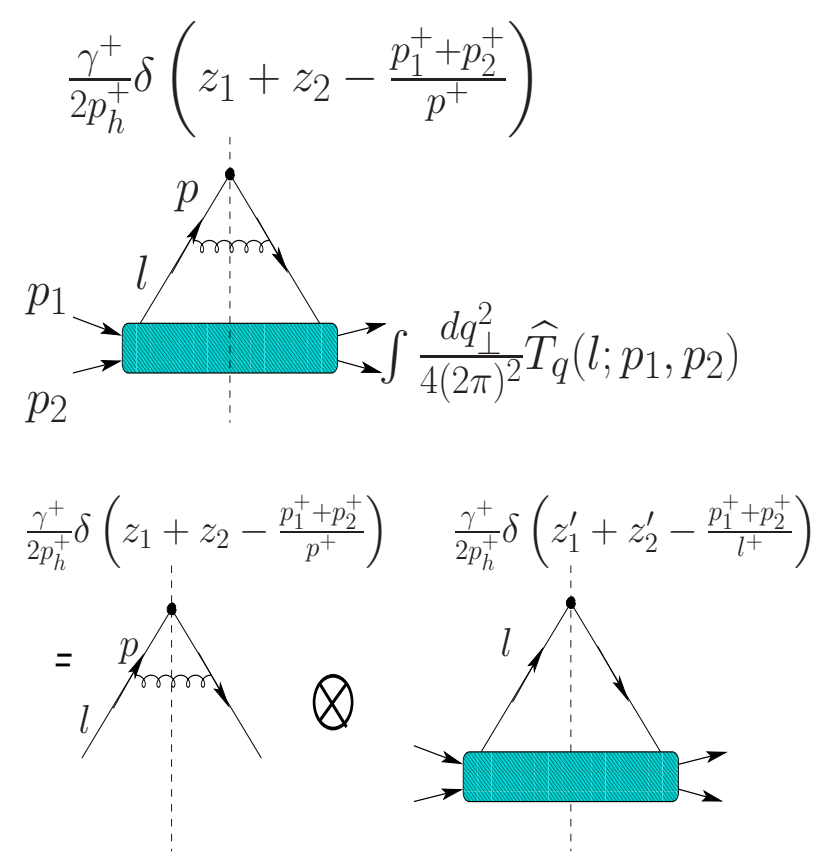

FIG. 7: Next-to-Leading order cut-vertex for quark fragmentation and its factorization.

terms of cut vertices. Inserting the hadronic tensor into the expression for the double differential cross section with minor simplifications, we obtain the factorized NLO contribution to the double inclusive cross section,

$$
\begin{aligned}
& \frac{d^{2} \sigma}{d z_{1} d z_{2}}=\sum_{q} \frac{4 \pi}{3} \frac{\alpha^{2} e_{q}^{2} N_{c}}{Q^{2}} \\
& \times \frac{\alpha_{s}}{2 \pi} \int_{\mu^{2}}^{Q^{2}} \frac{d p_{\perp}^{2}}{p_{\perp}^{2}} \int_{z}^{1} \frac{d y}{y^{2}} C_{F} \frac{1+y^{2}}{1-y} \\
& \frac{(z / y)^{4}}{4\left(z_{1} / y\right)\left(z_{2} / y\right)} \int \frac{d q_{\perp}^{2}}{8(2 \pi)^{2}} \int \frac{d^{4} l}{(2 \pi)^{4}} \delta\left(\frac{z}{y}-\frac{p_{h}^{+}}{l^{+}}\right) T_{q}\left(l ; p_{1}, p_{2}\right) .
\end{aligned}
$$

The last line of the above equation may be easily identified as $D\left(z_{1} / y, z_{2} / y, \mu^{2}\right)$, the dihadron fragmentation functions scaled up by the momentum fraction $y$, carried by the quark emanating from the split. Physically this represents the contribution to the fragmentation functions at a higher order brought about by gluon radiation carrying away with it a momentum fraction $1-y$. By itself this process displays both an infrared divergence as $y \rightarrow 1$ and a collinear divergence as $p_{\perp} \rightarrow 0$. The infrared divergence will be canceled by the virtual diagram contribution as shown in the next section. The collinear divergent part will be combined with the collinear diver- 
gent part of gluon fragmentation and absorbed into the renormalized fragmentation function.

\section{B. NLO contribution from gluon fragmentation}

We now proceed with the contribution from gluon fragmentation in the NLO processes. Essentially the outgoing hadronic state is replaced with the second line of Eq. 40]. Insertion of the interaction operator density followed by the contraction of the quark and antiquark operator with the out-going states $\left|k_{\bar{q}}\right\rangle$ and $\left|(p-l)_{q}\right\rangle$, leads to the following matrix element:

$$
\begin{aligned}
\mathcal{M}^{i i} & =i \sum_{q} \epsilon_{q} e^{2} g t^{a} \bar{v}_{k_{2}} \gamma^{\mu} u_{k_{1}} \frac{g_{\mu \nu}}{q^{2}+i \epsilon} \\
& \times\left\langle p_{1} p_{2} S-2\left|A_{\rho}^{a}(0)\right| 0\right\rangle \bar{u}^{r}(p-l)\left\{\frac{\gamma^{\rho} \boldsymbol{p} \gamma^{\nu}}{\mathbf{p}^{2}+i \epsilon}\right. \\
& \left.+\frac{\gamma^{\nu}(-\mathbf{k}-\eta)}{(\mathbf{k}+\mathbf{l})^{2}+i \epsilon}\right\} v^{s}(k)(2 \pi)^{4} \delta(\mathbf{q}-\mathbf{k}-\mathbf{p}-\mathbf{l}) .
\end{aligned}
$$

As in the previous subsection, the approximation of very high energies is made, allowing the isolation of the leading twist and leading log contribution of the corresponding hadronic tensor. This is obtained as

$$
\begin{aligned}
W^{\alpha \nu}= & \int \frac{d p_{1} d p_{2} d \cos \theta_{c m} d \phi_{c m} d \phi_{\perp}}{(2 \pi)^{4} 4 p_{1} p_{2}} \int \frac{d^{4} p}{(2 \pi)^{4}} \frac{d^{4} l}{(2 \pi)^{4}} \\
& 2 \pi \delta\left((\mathbf{q}-\mathbf{p})^{2}\right) 2 \pi \delta\left((\mathbf{p}-\mathbf{l})^{2}\right) g^{2} N_{c} C_{F} \hat{T}_{g}\left(l ; p_{1}, p_{2}\right) \\
\times & d_{\rho \sigma} \operatorname{Tr}\left[(\mathbf{p}-\boldsymbol{y}) \frac{\gamma^{\rho} \mathbf{k} \gamma^{\alpha}}{\mathbf{l}^{2}+i \epsilon}(\not \mathbf{q}-\not \mathbf{p}) \frac{\gamma^{\nu} \mathbf{l}^{\sigma}}{\mathbf{1}^{2}-i \epsilon}\right]
\end{aligned}
$$

where the gluon overlap matrix element $T_{g}\left(l ; p_{1}, p_{2}\right)$ is defined as

$$
\begin{aligned}
\hat{T}_{g}\left(l ; p_{1}, p_{2}\right)= & \int d^{4} x e^{i \mathbf{1} \cdot \mathbf{x}} \sum_{S-2} \\
& \int \frac{d q_{\perp}^{2}}{8(2 \pi)^{2}} \times\left\langle 0\left|A_{\mu}^{a}(x)\right| p_{1}, p_{2}, S-2\right\rangle \\
\times & \left\langle p_{1}, p_{2}, S-2\left|A_{\nu}^{b}(0)\right| 0\right\rangle \frac{\delta^{a b} d^{\mu \nu}}{16}
\end{aligned}
$$

A diagrammatical representation of this gluon fragmentation process can be illustrated as Fig. 8 The procedure leading to the extraction of the leading log and leading twist is rather similar to the case for the single fragmentation function and to the case of NLO process of quark fragmentation in the last subsection. Contracting the hadronic tensor with its leptonic counterpart we obtain the gluon contribution to the NLO double differ-

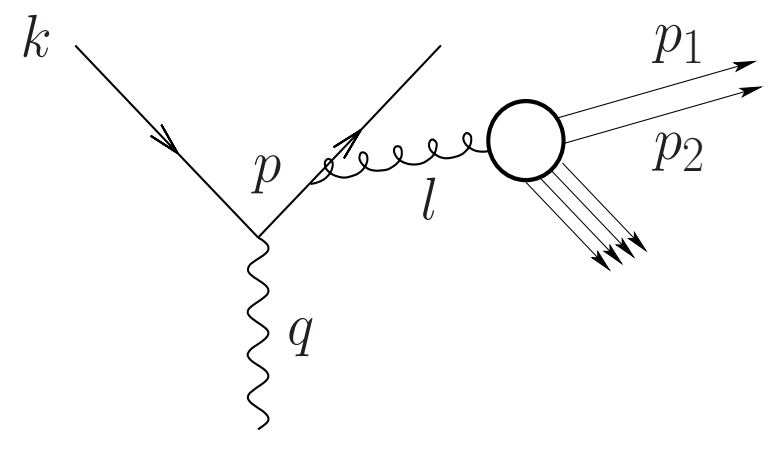

FIG. 8: The leading log gluon fragmentation contribution to the NLO modification of the quark fragmentation function.

ential cross section:

$$
\begin{aligned}
\frac{d^{2} \sigma}{d z_{1} d z_{2}}= & \sum_{q} \frac{4 \pi}{3} \frac{\alpha^{2} \epsilon_{q}^{2} N_{c}}{Q^{2}} \\
& \frac{\alpha_{s}}{2 \pi} \int_{\mu^{2}}^{Q^{2}} \frac{d p_{\perp}^{2}}{p_{\perp}^{2}} \int_{z}^{1} \frac{d y}{y^{2}} C_{F} \frac{1+(1-y)^{2}}{y} \\
& \frac{(z / y)^{3}}{2\left(z_{1} / y\right)\left(z_{2} / y\right)} \int \frac{d q_{\perp}^{2}}{8(2 \pi)^{2}} \int \frac{d^{4} l}{(2 \pi)^{4}} \\
& \times \delta\left(\frac{z}{y}-\frac{p_{h}^{+}}{l^{+}}\right) \hat{T}_{g}\left(l ; p_{1}, p_{2}\right) .
\end{aligned}
$$

It may come as no surprise that the above equation may also be derived from a set of Feynman rules involving cut-vertices. The cut-vertex diagrams are illustrated in Fig. 9 The rules are indicated in the figure. As a result of this computation we may now present the cut-vertex expression for the gluon dihadron fragmentation function (indicated in the lower right hand corner of Fig. $\mathrm{g}$ :

$$
\begin{aligned}
D_{g}\left(z_{1}, z_{2}\right) & =\frac{z^{3}}{2 z_{1} z_{2}} \int \frac{d q_{\perp}^{2}}{8(2 \pi)^{2}} \int \frac{d^{4} l}{(2 \pi)^{4}} \\
& \times \delta\left(z-\frac{p_{h}^{+}}{l^{+}}\right) \hat{T}_{g}\left(l ; p_{1}, p_{2}\right),
\end{aligned}
$$

where the factor $\hat{T}_{g}\left(l ; p_{1}, p_{2}\right)$ is given in Eq. 5.5. 


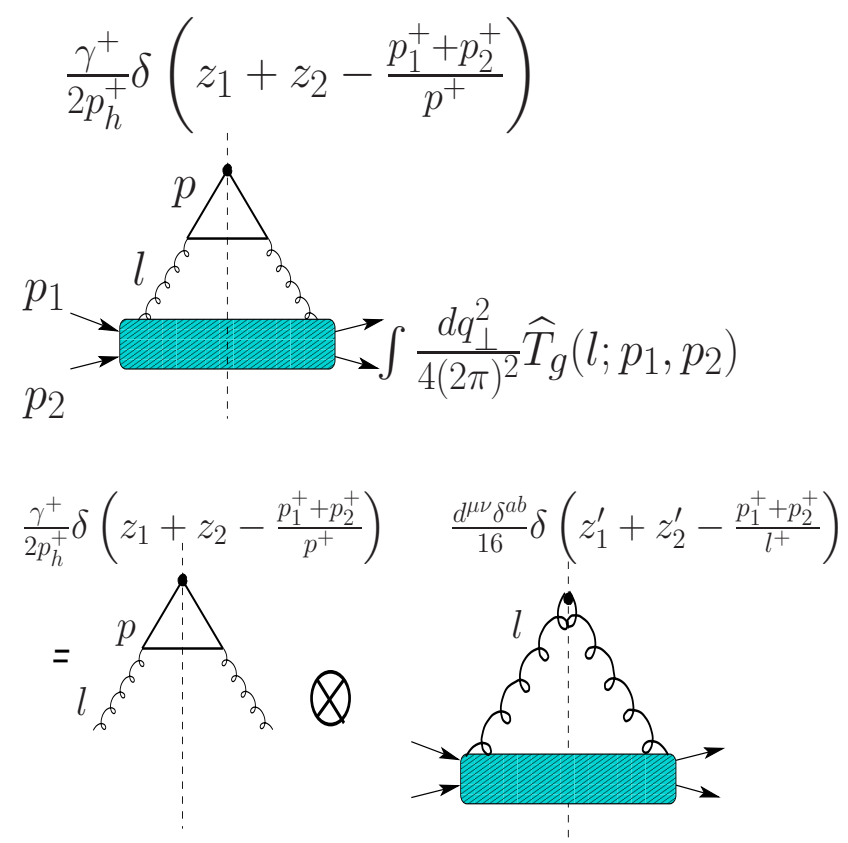

FIG. 9: Next-to-Leading order cut-vertex representation of the gluon fragmentation contribution.

\section{NLO contribution from quark and gluon single fragmentation}

In the previous subsections, we have evaluated two separate contributions to the NLO double fragmentation functions. In both of these we have assumed that the relative transverse momentum of the two detected hadrons is intrinsic and is limited by a scale $\mu_{\perp}$. In the definition of the dihadron fragmentation functions, the hadrons are detected with given fractions of forward longitudinal momentum but the transverse momenta are integrated over. Thus, all allowed transverse momenta between the detected hadrons must be included. In this paper, we will assume that all hadron pairs with relative transverse momentum larger than $\mu \gtrsim \mu_{\perp}$ are generated perturbatively. In the next-to-leading order, such hadron pairs can be produced from the independent fragmentation of the quark and gluon after their split, as illustrated in Fig. 10 Such a scenario has been considered in Refs. 1? 14, 15 where the double inclusive cross section with two detected hadrons in $e^{+} e^{-}$collisions with a fixed transverse momemtum between them was computed. The authors argued that in the case that the transverse momentum lies in a semihard region $\Lambda_{Q C D}<<q_{\perp}<<Q$, the dominant contribution to the cross section comes from the process where the fragmenting parton undergoes a semihard split into two independent partons which then fragment independently.

Under the condition that $q_{\perp}<<Q$ these hadrons can still be considered to belong to the same jet. Moreover, when $\Lambda_{Q C D}<<q_{\perp}$, the higher order contributions from multiple gluon vertex corrections to the semihard vertex are non-leading and thus the fragmentation of the two partons emanating from the split may be considered as independent. In Ref. 15, the authors demonstrated that the Sudakov double logarithms from the higher order vertex corrections are absent in the region $\Lambda_{Q C D}<<q_{\perp}$. In the interest of completeness we will repeat this derivation in a slightly different language in the Appendix.

We will evaluate the leading log and leading twist contribution in which the hadron pair comes from the independent fragmentation of the quark and gluon after a semi-hard split. We start again with the matrix element for this process,

$$
\begin{aligned}
\mathcal{M}^{i i i} & =i \sum_{q} e^{2} e_{q} g t^{a} \frac{g_{\nu \mu}}{q^{2}+i \epsilon} \bar{v}_{k_{1}} \gamma^{\mu} u_{k_{2}} \\
& \times\left\langle p_{1}, S_{1}-1\left|\bar{\psi}_{q}(0)\right| 0\right\rangle\left\langle p_{2}, S_{2}-1\left|A_{\rho}^{a}(0)\right| 0\right\rangle \\
& \times\left[\frac{\gamma^{\rho}(\mathbf{q}-\mathbf{k}) \boldsymbol{\gamma}^{\nu}}{(\mathbf{q}-\mathbf{k})^{2}+i \epsilon}+\frac{\gamma^{\nu}\left(\mathbf{p}_{S_{1}}-\boldsymbol{q}\right) \gamma^{\rho}}{\left(\mathbf{p}_{S_{1}}-\mathbf{q}\right)^{2}+i \epsilon}\right] v(k) \\
& \times(2 \pi)^{4} \delta^{4}\left(\mathbf{q}-\mathbf{p}_{S_{1}}-\mathbf{p}_{S_{2}}-\mathbf{k}\right) .
\end{aligned}
$$

The second term inside the square bracket corresponds to gluon emission from the antiquark in the quark direction and will not contribute in the leading log approximation. The out-state in this case is chosen to be the last line of Eq. 40. In this case, the sum over all final states of hadrons from the initial quark has been broken into two identical complete sets. In each of the sets, $S_{1}$ and $S_{2}$, a single hadron will be identified. Unlike in the previous two subsections, where one of the quark or gluon operators is contracted with a partonic state; both the quark and the gluon operators will be contracted with hadronic states in this case. The cross section constructed from this matrix element contains two separate sums over hadronic states; one of which has an overlap with a quark state, the other with a gluon state. The hadronic basis of states moving in the "away-side" jet will be replaced as before with the sum over all momentum states of a single antiquark:

$$
\sum_{S}=\frac{d^{3} p_{1}}{(2 \pi)^{3} 2 E_{1}} \frac{d^{3} p_{2}}{(2 \pi)^{3} 2 E_{2}} \frac{d^{3} k}{(2 \pi)^{3} 2 k} \sum_{S_{1}-1} \sum_{S_{2}-1} .
$$

In the last two subsections, the quark attached to the electromagnetic vertex is assigned the momentum $\mathbf{p}$, while the quark or gluon which materializes from the split is assigned the momentum 1 . In this subsection, the momentum of the fragmenting gluon and quark will be set to be $\mathbf{l}$ and $\mathbf{p}$. The quark attached to the electromagnetic vertex will thus have a momentum of $\mathbf{p}+\mathbf{1}$. This 
cosmetic reshuffling is solely to ease the extraction of the single fragmentation functions.

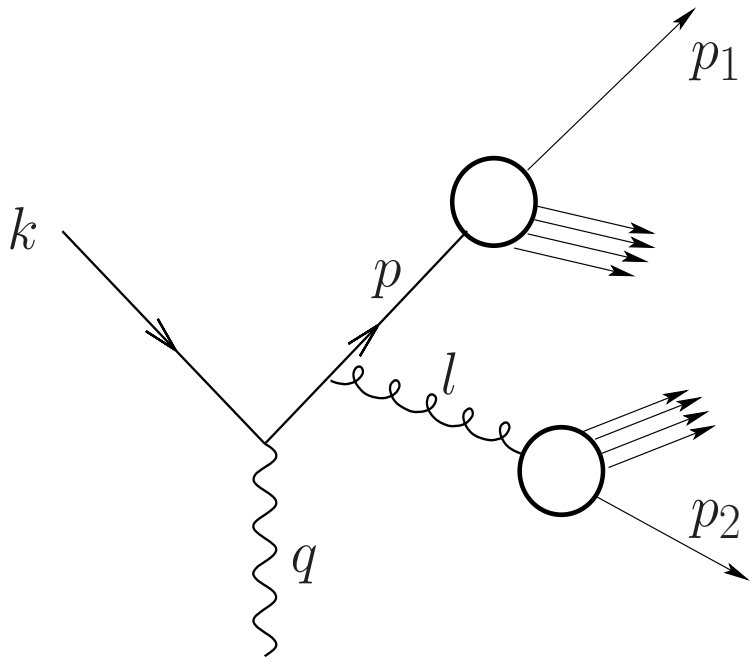

FIG. 10: The leading log mixed contribution to the NLO modification of the quark dihadron fragmentation function.

Again, we focus on the leading log portion of the matrix elements. This essentially restricts our attention to the square of the matrix element for the process depicted in Fig. 10] The integrated cross section for this process may be expressed as:

$$
\begin{aligned}
& \sigma^{i i i}=\frac{1}{2 s} \sum_{q} \int d^{4} y e^{i \mathbf{y} \cdot \mathbf{1}} e^{4} e_{q}^{2} g^{2} \frac{\mathcal{L}_{\nu \beta}}{4\left(\mathbf{q}^{2}\right)^{2}} N_{c} C_{F} \\
& \int \frac{d^{3} p_{1} d^{3} p_{2}}{(2 \pi)^{6} 4 p_{1} p_{2}} \frac{d^{4} k d^{4} l}{(2 \pi)^{8}} \delta^{+}\left(\mathbf{k}^{2}\right) \int d^{4} x e^{\mathbf{x} \cdot(\mathbf{q}-\mathbf{l}-\mathbf{k}-\mathbf{p})} e^{i \mathbf{x} \cdot \mathbf{p}} \\
& \sum_{S_{1}-1} \sum_{S_{2}-1} \operatorname{Tr}\left[\mathbf{k} \frac{\gamma^{\beta}(\boldsymbol{\phi} \mathbf{q}-\mathbf{k}) \gamma^{\nu}}{(\mathbf{q}-\mathbf{k})^{2}-i \epsilon}\right. \\
& \left.\times\left\langle 0\left|\psi_{q}(x)\right| p_{1}, S_{1}-1\right\rangle\left\langle S_{1}-1, p_{1}\left|\bar{\psi}_{q}(0)\right| 0\right\rangle \frac{\gamma^{\rho}(\boldsymbol{q}-\mathbf{k}) \gamma^{\nu}}{(\mathbf{q}-\mathbf{k})^{2}+i \epsilon}\right] \\
& \times \frac{\delta^{a b}}{2}\left\langle 0\left|A_{\sigma}^{b}(x)\right| p_{2}, S_{2}-1\right\rangle\left\langle S_{2}-1, p_{2}\left|A_{\rho}^{a}\right| 0\right\rangle,
\end{aligned}
$$

where two identities of unity

$$
1=\int d^{4} l \int \frac{d^{4} x}{(2 \pi)^{4}} \epsilon^{i \mathbf{1} \cdot \mathbf{x}},
$$

are inserted. As in the preceding subsections the standard shift of partonic variables is introduced i.e. $\mathbf{k} \rightarrow$ $\mathbf{q}-\mathbf{p}-\mathbf{l}$ and $\int d^{4} k \rightarrow \int d^{4} p$. The squares of the overlap matrix elements between the partonic operators $\psi, A_{\rho}^{a}$ and the hadronic states will result in the single fragmentation functions. Absorbing the integrals over undetected hadron states and the Fourier integrals, the $\hat{T}$ matrix elements may be written as (see Sec. II or Ref. 21):

$$
\begin{aligned}
& {\left[\hat{T}_{g}\right]_{\sigma \rho}^{b a}=\int d^{4} y e^{\mathbf{y} \cdot \mathbf{l}} \sum_{S_{2}-1}} \\
& \quad\left\langle 0\left|A_{\sigma}^{b}(y)\right| S_{2}-1, p_{2}\right\rangle\left\langle p_{2}, S_{2}-1\left|A_{\rho}^{a}(0)\right| 0\right\rangle,
\end{aligned}
$$

which leads to the definition of the gluon fragmentation function at leading twist. The same extraction may also be performed for the quark overlap matrix operator:

$$
\begin{aligned}
& {\left[\hat{T}_{q}\right]^{\alpha \gamma}=\int d^{4} x e^{\mathbf{x} \cdot \mathbf{p}} \sum_{S_{1}-1}} \\
& \left\langle 0\left|\psi_{q}^{\alpha}(x)\right| S_{1}-1, p_{1}\right\rangle\left\langle p_{1}, S_{2}-1\left|\psi_{q}^{\gamma}(0)\right| 0\right\rangle,
\end{aligned}
$$

which leads to the definition of the quark fragmentation function at leading order. Within the collinear approximation applied to the above two matrix elements, they may be approximated at leading twist as

$$
\begin{aligned}
{\left[\hat{T}_{g}\right]_{\sigma \rho}^{b a}\left(l ; p_{2}\right) } & \simeq \delta^{a b} d_{\sigma \rho}(l) T_{g}\left(l ; p_{2}\right), \\
\hat{T}_{q}\left(p ; p_{1}\right) & \simeq \frac{\boldsymbol{p}_{1}}{2} T_{q}\left(p ; p_{1}\right) .
\end{aligned}
$$

With the definition of the single fragmentation functions and the collinear approximation, the cross section for independent fragmentation may be expressed in a simplified form as

$$
\begin{aligned}
& \sigma^{i i i}=\frac{1}{2 s} \sum_{q} \frac{\epsilon^{4} e_{q}^{2} N_{c}}{4 Q^{4}} \mathcal{L}_{\beta \nu} g^{2} C_{F} \int \frac{d^{3} p_{1} d^{3} p_{2}}{(2 \pi)^{6} 4 E_{1} E_{2}} \\
& \int \frac{d^{4} l d^{4} p}{(2 \pi)^{8}} 2 \pi \delta^{+}\left((\mathbf{q}-\mathbf{l}-\mathbf{p})^{2}\right) T_{g}\left(l ; p_{2}\right) T_{q}\left(p ; p_{1}\right) d_{\rho \sigma}(l) \\
& \times \operatorname{Tr}\left[(\not \mathbf{q}-\boldsymbol{p}-\not \mathbf{p}) \gamma^{\beta}\left\{\frac{\not+\not{p}}{(\mathbf{l}+\mathbf{p})^{2}} \gamma^{\sigma} \frac{\not \boldsymbol{p}_{1}}{2} \gamma^{\rho} \frac{\boldsymbol{\gamma}+\not{p}}{(\mathbf{l}+\mathbf{p})^{2}}\right\} \gamma^{\nu}\right] .(65)
\end{aligned}
$$

This is the leading log contribution to the inclusive cross section for the production of two identified hadrons at next-to-leading-order where each hadron emanates from the independent fragmentation of a parton. The overlap matrix elements which lead to the definition of the fragmentation functions, $T_{g}$ and $T_{q}$ have already been factored out. This represents a NLO contribution to the double fragmentation of the quark emanating from the electromagnetic vertex.

Again, we use a collinear approximation to isolate the leading twist part of the terms inside the curly brackets:

$$
\begin{aligned}
& \frac{\not+\not \mathbf{p}}{(\mathbf{l}+\mathbf{p})^{2}} \gamma^{\sigma} \frac{\not \hat{p}_{1}}{2} \gamma^{\rho} \frac{\not 1+\not \mathfrak{p}}{(\mathbf{l}+\mathbf{p})^{2}} d_{\rho \sigma}(l) \\
& \simeq \frac{\mathbf{p}_{h}}{2} \operatorname{Tr}\left[\frac{\gamma^{+}}{2 p_{h}^{+}} \frac{\not+\not \mathbf{p}}{(\mathbf{l}+\mathbf{p})^{2}} \gamma^{\sigma} \frac{\mathbf{p}_{1}}{2} \gamma^{\rho} \frac{\not+\not \mathbf{p}}{(\mathbf{l}+\mathbf{p})^{2}} d_{\rho \sigma}(l)\right] \text {. }
\end{aligned}
$$


After introducing two momentum fractions $z_{1}^{\prime}$ and $z_{2}^{\prime}$ through a multiplicative factor of unity,

$$
1=\int_{0}^{1} d z_{1}^{\prime} d z_{2}^{\prime} \delta\left(z_{1}^{\prime}-\frac{p_{1}^{+}}{p^{+}}\right) \delta\left(z_{2}^{\prime}-\frac{p_{2}^{+}}{l^{+}}\right),
$$

and a rearrangement of the integrals we have the following factorized from of the cross section at leading twist,

$$
\begin{aligned}
& \sigma^{i i i}=\frac{1}{2 s} \sum_{q} \frac{e^{4} \epsilon_{q}^{2} N_{c}}{4 Q^{4}} \mathcal{L}_{\beta \nu} g^{2} C_{F} \int \frac{d z_{1}^{\prime} d z_{2}^{\prime} d^{3} p_{1} d^{3} p_{2}}{(2 \pi)^{6} 4 E_{1} E_{2}} \\
& \times\left.\operatorname{Tr}\left[(\not \mathbf{q}-\mathbf{y}-\not \mathbf{p}) \gamma^{\beta} \frac{\mathbf{p}_{h}}{2} \gamma^{\nu}\right] 2 \pi \delta^{+}\left((\mathbf{q}-\mathbf{l}-\mathbf{p})^{2}\right)\right|_{\mathbf{p}=\mathbf{p}_{1} / z_{1}^{\prime}} ^{\mathbf{l}=\mathbf{p}_{2} / z_{2}^{\prime}} \\
& \times\left[d_{\rho \sigma}(l) \operatorname{Tr}\left\{\frac{\gamma^{+}}{2 p_{h}^{+}} \frac{\mathbf{y}+\not{p}}{(\mathbf{l}+\mathbf{p})^{2}} \gamma^{\sigma} \frac{\not \mathbf{p}_{1}}{2} \gamma^{\rho} \frac{\mathbf{y}+\not{p}}{(\mathbf{l}+\mathbf{p})^{2}}\right\}\right]_{\mathbf{p}=\mathbf{p}_{1} / z_{1}^{\prime}}^{\mathbf{l}=\mathbf{p}_{2} / z_{2}^{\prime}} \\
& \times \int \frac{d^{4} l}{(2 \pi)^{4}} \delta\left(z_{2}^{\prime}-\frac{p_{2}^{+}}{l^{+}}\right) T_{g}\left(l ; p_{2}\right) \\
& \times \int \frac{d^{4} p}{(2 \pi)^{4}} \delta\left(z_{1}^{\prime}-\frac{p_{1}^{+}}{p^{+}}\right) T_{q}\left(p ; p_{1}\right) .
\end{aligned}
$$

It is apparent that the second line of the above equation corresponds to the hard cross section of an $e^{+} e^{-}$pair annihilating via a single virtual photon to a $q \bar{q}$ pair. The third line corresponds to the splitting of the quark into a quark and gluon. Note the absence of any $\delta$-function maintaining an on-shell condition. This indicates that neither the quark nor gluon is being cut. The fourth and fifth line indicate the independent fragmentation of the quark and gluon into hadrons with the identification of a single hadron from each of these sources. The cutvertex structure of this process resembles that of Fig. [I] The trace over the Dirac matrix structure of the third line may be performed, followed by a contraction of the Lorentz indices to obtain:

$$
\frac{z_{1}^{\prime} 8\left(p_{1}^{+} / z_{1}^{\prime}+p_{2}^{+} / z_{2}^{\prime}\right)}{4 p_{h}^{+}\left(\mathbf{p}_{1} / z_{1}^{\prime}+\mathbf{p}_{2} / z_{2}^{\prime}\right)^{2}} \frac{1+y^{2}}{1-y},
$$

where the variable $y$ is introduced once again as the integral over a $\delta$-function

$$
\int_{0}^{1} d y \delta\left(y-\frac{p_{1}^{+} / z_{1}^{\prime}}{p_{1}^{+} / z_{1}^{\prime}+p_{2}^{+} / z_{2}^{\prime}}\right)
$$

One notes that the variable $y$ is essentially the ratio of the forward light-cone momentum of the offspring quark to that of the parent. This leads to the same splitting function as that of a quark splitting to a quark and a gluon. We may have chosen $y$ to represent the ratio of the energy of the gluon to that of the parent quark. This would have resulted in a splitting function similar to that of the preceding subsection. Changing the order of integration between the various ratios $y, z_{1}^{\prime}, z_{2}^{\prime}$, we define the quantities $z_{1}=z_{1}^{\prime} / y$ and $z_{2}=z_{2}^{\prime} /(1-y)$. Note that the double differential cross section $d^{2} \sigma / d z_{1} d z_{2}$ involves the ratios $z_{1}, z_{2}$ of the hadronic forward light-cone momentum to that of the parent quark emanating from the electromagnetic vertex. Following this we may again switch the order of integration,

$$
\begin{aligned}
& \int_{0}^{1} d z_{1}^{\prime} \int_{0}^{1} d z_{2}^{\prime} \int_{0}^{1} d y \\
= & \int_{0}^{1} d y \int_{0}^{1} d z_{1}^{\prime} \int_{0}^{1} d z_{2}^{\prime} \\
= & \int_{0}^{1} d y \int_{0}^{1 / y} \frac{d z_{1}}{y} \int_{0}^{1 /(1-y)} \frac{d z_{2}}{(1-y)} \\
= & \int_{0}^{1} d z_{1} \int_{0}^{1} d z_{2} \int_{z_{1}}^{1-z_{2}} \frac{d y}{y(1-y)}
\end{aligned}
$$

The integration over the hadronic momenta may now be subjected to the same variable transformation as demonstrated in Fig. 4 Within the collinear approximation it may be easily demonstrated that

$$
(\mathbf{l}+\mathbf{p})^{2}=\frac{q_{\perp}^{2} p_{h}^{+2} y(1-y)}{4 p_{1}^{+} p_{2}^{+} z_{1} z_{2}}
$$

The $\delta$-function introduced in Eq. 69 may also be similarly simplified to obtain,

$$
\begin{aligned}
\delta\left(y-\frac{p_{1}^{+} / z_{1}^{\prime}}{p_{1}^{+} / z_{1}^{\prime}+p_{2}^{+} / z_{2}^{\prime}}\right) & =\delta\left[\frac{y(1-y)}{p^{+}+l^{+}}\left(\frac{p_{2}^{+}}{z_{2}}-\frac{p_{1}^{+}}{z_{1}}\right)\right] \\
& =\frac{p^{+}+l^{+}}{y(1-y)} \delta\left(\frac{p_{2}^{+}}{z_{2}}-\frac{p_{1}^{+}}{z_{1}}\right)(72)
\end{aligned}
$$

where the quantities $p^{+}$and $l^{+}$are subjected to the condition of constraint introduced in Eq. 68. The $\delta$-function is now similar to the second $\delta$-function in Eq. 3.5. and may be used to extract the hard cross section $\sigma_{0}^{q q}$ [see Eq. [2]].

In the last two subsections, there is an integration over the transverse momentum of the parent quark emanating from the EM vertex. This integration has a collinear divergence that must be absorbed into the renormalized fragmentation functions. The light-like null vector $\mathbf{n}$ was alligned in a direction such that its three-components remained opposite to those of $\mathbf{p}_{h}$, the sum of the momenta of the detected hadrons. By definition $\mathbf{n}$ has no transverse component. As both hadrons originated from the same parton, the preceding condition along with the assumption of collinearity of the final hadrons with the fragmenting parton, constrained the transverse component of the fragmenting parton to be near vanishing. In this case we have again chosen the three components of $\mathbf{n}$ 

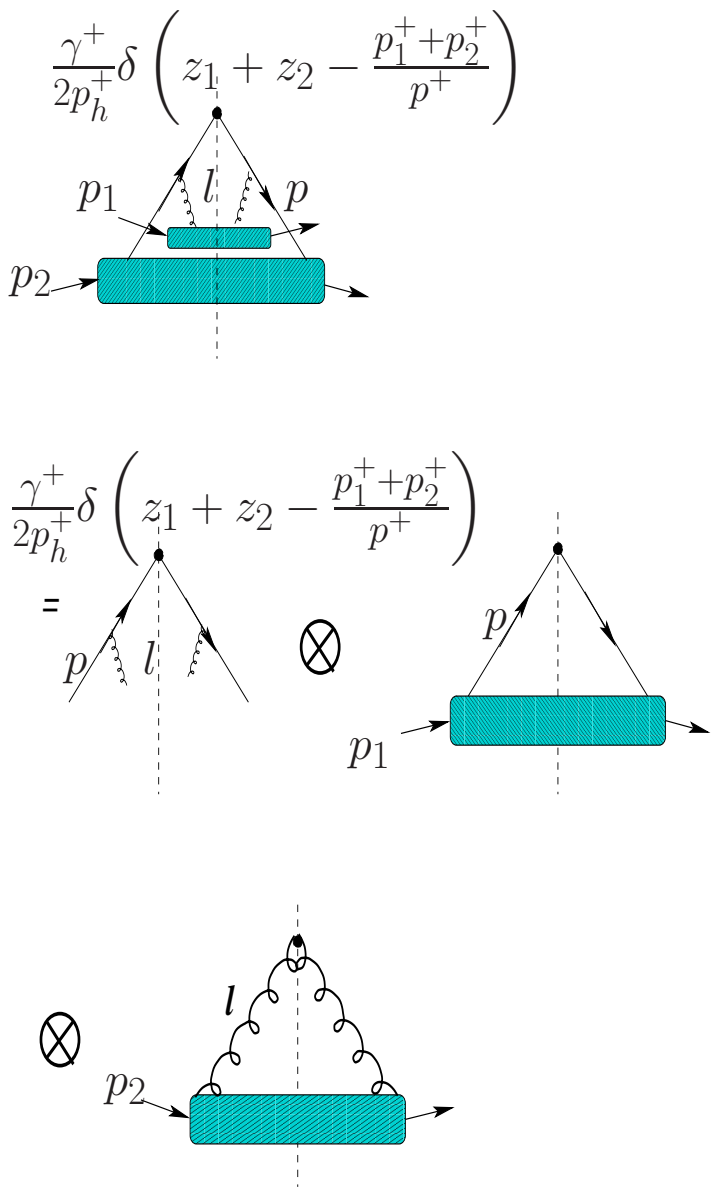

FIG. 11: Next-to-Leading order cut-vertex representation of the mixed contribution.

to be opposite to those of $\mathbf{p}_{h}$. However, the two detected hadrons in this subsection emerge from two different partons which come from a split of the parent quark. As a result, whereas both fragmenting partons have a transverse component $\left(l_{\perp}, p_{\perp}\right)$ proportional to the transverse component of the detected hadrons $q_{\perp}$, the parent quark is restricted to near vanishing values of a transverse component. In conformity with the previous subsections and with the DGLAP evolution of the single fragmentation function, the integration over a transverse component will remain that of a partonic momentum. The hadronic transverse component $q_{\perp}$ may be trivially related to its partonic counterpart thus:

$$
q_{\perp}=2 l_{\perp} \frac{z_{1} z_{2}}{y(1-y)\left(z_{1}+z_{2}\right)}
$$

With these approximations, it may be easily demonstrated that the double differential cross section of independent fragmentation assumes the form as sketched in the concluding lines of Eq. 5 , i.e.,

$$
\begin{aligned}
\frac{d^{2} \sigma^{i i i}}{d z_{1} d z_{2}}= & \sum_{q} \sigma_{0}^{q \bar{q}} \frac{\alpha_{s}}{2 \pi} \int \frac{d l_{\perp}^{2}}{l_{\perp}^{2}} \int_{z_{1}}^{1-z_{2}} \frac{d y}{y(1-y)} \\
& C_{F} \frac{1+y^{2}}{1-y} D_{q}\left(\frac{z_{1}}{y}\right) D_{g}\left(\frac{z_{2}}{1-y}\right) .
\end{aligned}
$$

Where $D_{q}$ and $D_{g}$ are the single fragmentation functions of a quark and a gluon 8 (see also Sect. II).

The careful reader will have noted that the order of integration between $y$ and $l_{\perp}$ has been switched; whereas from Eq. 73 we see that if $q_{\perp}$ is independent of $y$ then $l_{\perp}$ is a function of $y$. There are two ways to resolve this. We may ascribe a multiplicative $y$ dependence to the maximum value of $q_{\perp}$. Indeed this would be an artificial dependence, and would physically mean the inclusion of an extra piece of phase space in the $l_{\perp}$ integration. Any such multiplicative factor would have no influence on the DGLAP evolution equations as these would involve a differentiation with respect to $Q^{2}$ i.e.

$$
\frac{\partial}{\partial Q^{2}} \int^{\delta^{2} Q^{2}} \frac{d l_{\perp}^{2}}{l_{\perp}^{2}}=\frac{\partial}{\partial Q^{2}} \int^{Q^{2}} \frac{d l_{\perp}^{2}}{l_{\perp}^{2}}
$$

Yet another approach may be to view the order of integrals in Eq. Ful as merely symbolic, the differentiation with respect to $Q^{2}$ leads to the same evolution equations.

There remains one last contribution to the inclusive cross section for the production of two hadrons from a parent quark: this is obtained trivially by switching $z_{1}$ and $z_{2}$ in Eq. 741, i.e. the hadron with momentum fraction $z_{1}$ originates from the fragmentation of the gluon rather than the quark. With the addition of the above mentioned contribution, we complete the discussion of the real radiative corrections to the inclusive cross section of same-side dihadron production. The contributions discussed in this subsection possess no infrared divergence as the $y$ integration is terminated at $1-z_{2}$ or $1-z_{1}$, contrary to dihadron fragmentation from the single quark whose infrared divergence is cancelled by the virtual correction.

\section{RENORMALIZED FRAGMENTATION FUNCTIONS AND DGLAP EVOLUTION}

So far we have calculated the real radiative corrections to the dihadron fragmentation functions. One notices that the contribution from quark fragmentation after a gluon radiation in Eq. $5 \%$ contains an infrared divergence. Such an infrared divergence will be canceled by virtual contributions from interference diagrams such as those of Fig. [? It is well known that in gauge theories, in light-cone gauge, the leading log contribution is contained solely within the self-energy diagram and not shared between the self-energy and the vertex correction, 
as is the case in the Feynman gauge 8 . The hadronic tensor for such a virtual correction is

$$
\begin{aligned}
& W^{\mu \nu}=\sum_{S-2} \int \frac{d^{3} p_{1} d^{3} p_{2}}{(2 \pi)^{6} 4 E_{1} E_{2}} \frac{d^{4} p}{(2 \pi)^{4}} 2 \pi \delta\left((\mathbf{q}-\mathbf{p})^{2}\right) \\
& \times \operatorname{Tr}\left[\widehat{T}_{q}\left(p ; p_{1}, p_{2}\right)\{-i \Sigma(p)\} \frac{i}{\not \mathbf{p}} \gamma^{\nu}(\not \mathbf{q}-\not{p}) \gamma^{\mu}\right],
\end{aligned}
$$

where, $-i \Sigma(p)$ respresents the one-loop quark self-energy of a quark with four-momentum $\mathbf{p}$.

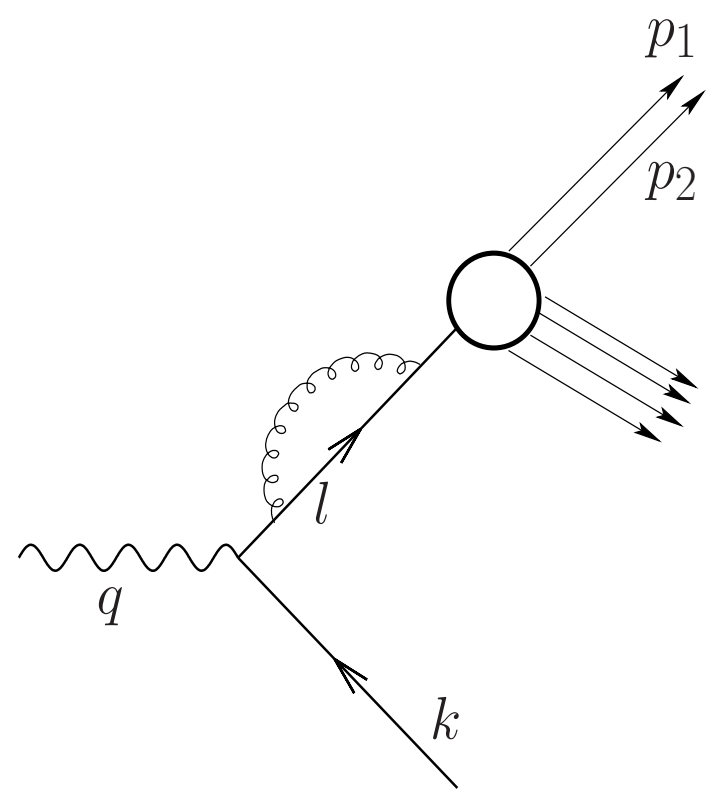

FIG. 12: The leading log self-energy contribution to the NLO modification of the quark fragmentation function.

The hadronic tensor may again be factorized at leading twist following much the same procedure as those of the last section. There remains the integration over the internal gluon momentum 1 . The leading behaviour of this integral, as in the case of single fragmentation, in the part of phase space that includes a pinch singularity on the $l^{-}$contour, and endpoint singularities on the $l_{\perp}$ and $l^{+}$contours. The pinch singlularity may occur only in the region where

$$
0<l^{+}<p^{+} \quad \text { and } \quad 0<l_{\perp}^{2}<\mathbf{p}^{2} \leq Q^{2} .
$$

The derivation of the leading behaviour, which mostly mirrors the calculation for single fragmentation functions, will not be presented in full detail here. We refer the reader to Ref. 24 , for details. The final result of the self-energy correction to the partial double differential cross section is

$$
\begin{aligned}
\frac{d^{2} \sigma}{d z_{1} d z_{2}} & =-\sum_{q} \sigma_{0}^{q \bar{q}} \frac{\alpha_{s}}{2 \pi} \int^{Q^{2}} \frac{d l_{\perp}^{2}}{l_{\perp}^{2}} \\
& \times \int_{0}^{1} \frac{1+y^{2}}{1-y} D_{q}^{h_{1} h_{2}}\left(z_{1}, z_{2}\right) .
\end{aligned}
$$

Note that the transverse integration is over $l_{\perp}$ and not the parent quark momenta. The variable $y$ is defined as $y=l^{+} / p^{+}$. The overall negative sign should not be a cause for alarm, as this is only a part of the total cross section. Combining the above equation with Eq. 52 leads to the cancellation of the infrared singularity as $y \rightarrow 1$. One can effectively combine the virtual and real corrections with a "+"-function,

$$
\begin{aligned}
P_{q \rightarrow q g}(y) & =C_{f}\left[\frac{1+y^{2}}{(1-y)}\right]_{+} \\
& =C_{f}\left[\frac{1+y^{2}}{(1-y)_{+}}+\frac{3}{2} \delta(1-y)\right],
\end{aligned}
$$

where the "+"-function is defined as

$$
\int_{0}^{1} d y \frac{F(y)}{(1-y)_{+}} \equiv \int_{0}^{1} d y \frac{F(y)-F(1)}{1-y}
$$

with $F(y)$ being any function that is sufficiently smooth at $y=1$.

In the remaining, we will focus on the non-singlet (NS) fragmentation functions for simplicity. In this case, the contribution from dihadron gluon fragmentation drops out. Summing all three types of contributions from the last section, we obtain the NLO contribution to the NS dihadron fragmentation function,

$$
\begin{aligned}
& D_{N S}^{h_{1}, h_{2}}\left(z_{1}, z_{2}, Q^{2}\right)=D_{N S}^{h_{1}, h_{2}}\left(z_{1}, z_{2}\right) \\
& \quad+\frac{\alpha_{s}}{2 \pi} \int^{Q^{2}} \frac{d p_{\perp}^{2}}{p_{\perp}^{2}} \int_{z}^{1} \frac{d y}{y^{2}} C_{F}\left(\frac{1+y^{2}}{1-y}\right)_{+} D_{N S}^{h_{1}, h_{2}}\left(\frac{z_{1}}{y} ; \frac{z_{2}}{y}\right) \\
& \quad+\frac{\alpha_{s}}{2 \pi} \int^{Q^{2}} \frac{d l_{\perp}^{2}}{l_{\perp}^{2}} \int_{z_{1}}^{1-z_{2}} \frac{d y}{y(1-y)} \\
& \quad \times C_{F} \frac{1+y^{2}}{1-y} D_{N S}^{h_{1}}\left(\frac{z_{1}}{y}\right) D_{g}^{h_{2}}\left(\frac{z_{2}}{1-y}\right)
\end{aligned}
$$

where the leading order fragmentation functions are defined as matrix elements of field operators in Eq. 37 and 18 . This has exactly the same structure as we have outlined in the parton model in Eq. 7. Therefore, the definition of renormalized fragmentation functions and the derivation of the DGLAP evolution can be similarly applied here.

The renormalized dihadron fragmentation function is defined as 


$$
\begin{aligned}
& D_{N S}^{h_{1}, h_{2}}\left(z_{1}, z_{2}, \mu^{2}\right) \equiv D_{N S}^{h_{1}, h_{2}}\left(z_{1}, z_{2}\right) \\
& +\frac{\alpha_{s}}{2 \pi} \int^{\mu^{2}} \frac{d p_{\perp}^{2}}{p_{\perp}^{2}} P_{q \rightarrow q g} * D_{N S}^{h_{1} h_{2}} \\
& \quad+\frac{\alpha_{s}}{2 \pi} \int^{\mu^{2}} \frac{d l_{\perp}^{2}}{l_{\perp}^{2}} \hat{P}_{q \rightarrow q 9} \bar{*}\left[D_{N S}^{h_{1}} D_{g}^{h_{2}}\right]
\end{aligned}
$$

We point out, once again, that the scale $\mu$ at which the renormalized functions are defined is chosen above the semihard scale $\mu_{\perp}$. At this scale, corrections to the renormalized quantities may be evaluated perturbatively. In terms of the renormalized dihadron fragmentation function, the dihadron fragmentation function at NLO can be expressed as

$$
\begin{aligned}
D_{N S}^{h_{1}, h_{2}}\left(z_{1}, z_{2}, Q^{2}\right) \equiv D_{N S}^{h_{1}, h_{2}}\left(z_{1}, z_{2}, \mu^{2}\right) \\
+\frac{\alpha_{s}}{2 \pi} \log \left(\frac{Q^{2}}{\mu^{2}}\right) P_{q \rightarrow q g} * D_{N S}^{h_{1} h_{2}}\left(\mu^{2}\right) \\
+\frac{\alpha_{s}}{2 \pi} \log \left(\frac{Q^{2}}{\mu^{2}}\right) \hat{P}_{q \rightarrow q g} \bar{*}\left[D_{N S}^{h_{1}}\left(\mu^{2}\right) D_{g}^{h_{2}}\left(\mu^{2}\right)\right],
\end{aligned}
$$

where we have also used the renormalized form of the single fragmentation functions.

Note that we have introduced a minimum limit to the scale $\mu \geq \mu_{\perp}$ in the definition of the renormalized dihadron fragmentation function. If one insisted in choosing $\mu<\mu_{\perp}$, then the contribution from independent quark and gluon fragmentation after a semi-hard split would have to be factorized off at the scale $\mu_{\perp}$. In other words the minimum of the second logarithm in Eq. $\mathbf{8 *}$ would be set at $\mu_{\perp}^{2}$ and would only contribute in the event that $Q^{2}$ is chosen greater than $\mu_{\perp}^{2}$. In this way, we have assumed that only hadron pairs with relative transverse momentum $q_{\perp}>\mu_{\perp}$ are generated perturbatively from independent fragmentation of two separate partons in the process of a perturbative cascade. For two partons whose relative transverse momentum is smaller than $\mu_{\perp}$, nonperturbative processes become important and their fragmentation cannot be independent anymore. We include this part, which also contains a collinear divergence, in the renormalized dihadron fragmentation function. This non-perturbative scale can also be considered as the intrinsic relative transverse momentum of the dihadron fragmentation function and it should set the limit of the integration over $q_{\perp}$ in the matrix element definition of the dihadron fragmentation function in Eq. 37. If one wants to consider the unintegrated (over $q_{\perp}$ ) dihadron fragmentation function, $\mu_{\perp}$ could set the initial condition for the $q_{\perp}$ distribution and can be used to study the evolution equation of the angular distribution inside a jet. For now, this scale will only set a limit of the physical scale $Q^{2}>>\mu^{2}>\mu_{\perp}^{2}>>\Lambda_{Q C D}^{2}$ for the DGLAP evolution and will not enter the equation. In the ensuing calculation of the evolution of the fragmentation functions we will not enter into such subtleties and always chose the starting scale $\mu \gtrsim \mu_{\perp}$.
To include the entire leading log modification, contributions from all the diagrams outlined in Fig. I have to be resummed into the scale dependent fragmentation functions. These are then differentiated to obtain the evolution equation which is given exactly as in Eq. 15 for NS dihadron fragmentation function.

\section{NUMERICAL RESULTS OF NON-SINGLET EVOLUTION}

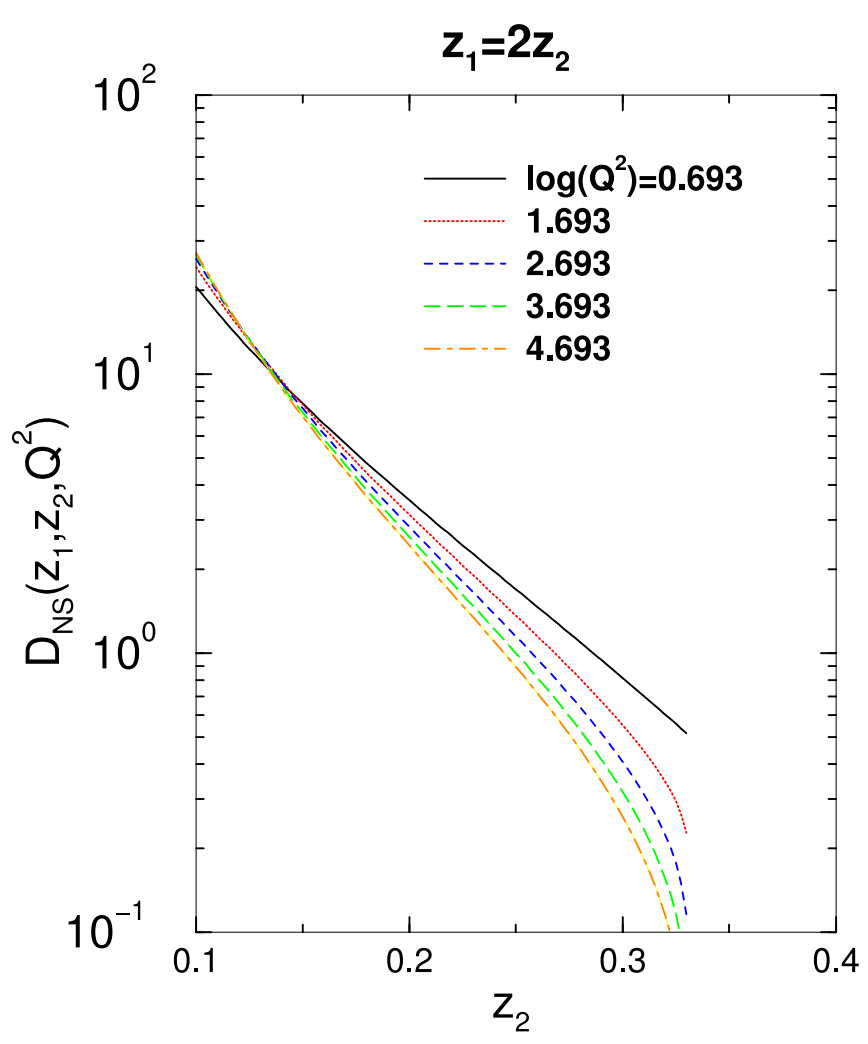

FIG. 13: Results of the evolution of the non-singlet quark dihadron fragmention function $D_{q}^{h_{1} h_{2}}\left(z_{1}, z_{2}\right)$, where $z_{1}=2 z_{2}$, from $Q^{2}=2 \mathrm{GeV}^{2}$ to $109 \mathrm{GeV}^{2}$. See text for details.

In this section we will study numerically the DGLAP evolution of the non-singlet dihadron fragmentation. As in many other cases of DGLAP evolution, the solutions require an initial condition of the fragmentation functions at an initial scale. Such initial conditions, as in the case of single fragmentation functions, are non-perturbative and are usually constructed from the experimental measurement of the single inclusive differential cross section $d \sigma / d z$, according to Eq. II at LO. The evolution of the fragmentation functions with the energy scale of the reaction can then be calculated from the DGLAP equations.

The absence of any experimental data for two particle correlation in $e^{+} e^{-}$annihilation forces us to formulate an ansatz of the initial condition. We simply use it as a toy model to illustrate the DGLAP evolution of the dihadron fragmentation functions. We take the LO product of two 


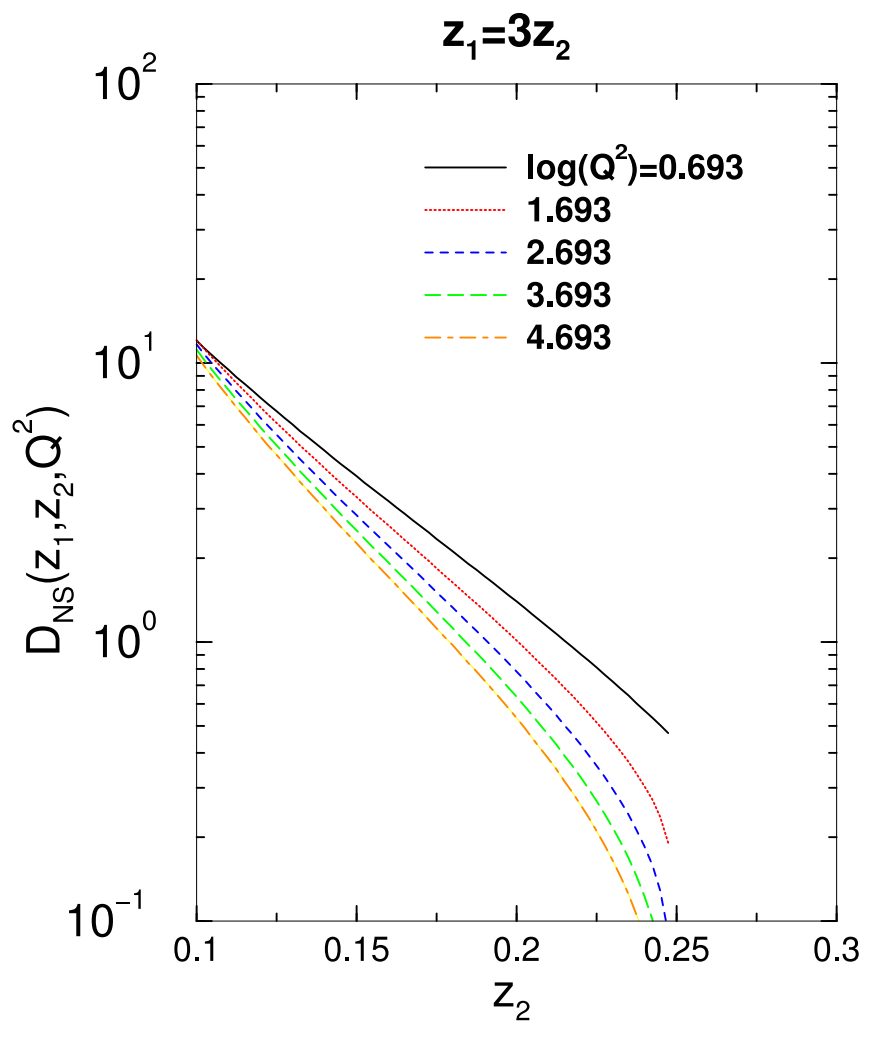

FIG. 14: Same as Fig. 13 except $z_{1}=3 z_{2}$.

single fragmentation functions as the initial condition for the evolution of the fragmentation function, i.e.,

$$
\begin{aligned}
D_{q}^{h_{1} h_{2}}\left(z_{1}, z_{2}, \mu^{2}\right) & =D_{q}^{h_{1}}\left(z_{1}, \mu^{2}\right) D_{q}^{h_{2}}\left(z_{2}, \mu^{2}\right) \\
& \times \theta\left(1-z_{1}-z_{2}\right) .
\end{aligned}
$$

We set the initial condition at $Q^{2}=2 \mathrm{GeV}^{2}$. This corresponds to $\log Q^{2}=0.693$. While it may be argued that the initial energy is somewhat low for the applicability of pQCD in $e^{+} e^{-}$annihilation, we consider it as just the scale of the momentum transfer while the actual jet energy could be sufficiently high. The differential equation corresponding to $\mathrm{Eq}$. $\mathbf{4 5}$ is then solved by the simple methods of a second order Runge-Kutta numerical estimation. Results are presented in Figs. 1320 at intervals of $\Delta \log Q^{2}=1.0$ The initial condition is represented by the solid black line in all plots. We stop the evolution at $\log Q^{2}=4.693$, which corresponds to $Q^{2} \simeq 109 \mathrm{GeV}^{2}$.

We present results where the leading particle possesses a multiple of the momentum fraction of the next-toleading particle. We begin with plots of just the evolution of the non-singlet dihadron fragmentation function at $z_{1}=2 z_{2}$ in Fig. I3 and $z_{1}=3 z_{2}$ in Fig. I4 In these and all other plots the results are always presented as a function of $z_{2}$. The results of the evolution are not qualitatively different from those of the single fragmentation function. Since the sum of the momentum fractions are constrained to unity i.e. $z_{1}+z_{2} \leq 1$, we find that

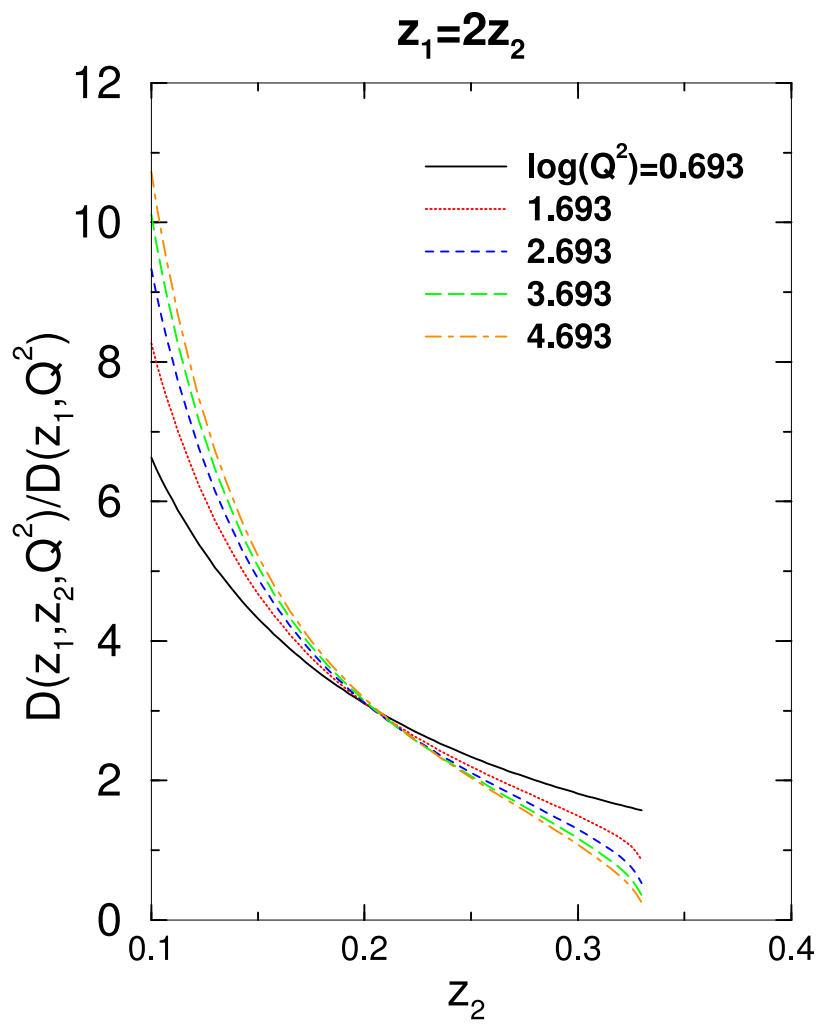

FIG. 15: Results of the ratio of the non-singlet quark dihadron fragmention function $D_{q}^{h_{1} h_{2}}\left(z_{1}, z_{2}, Q^{2}\right)$ to the single leading fragmentation function $D_{q}^{h_{1}}\left(z_{1}, Q^{2}\right)$. In this case $z_{1}=2 z_{2}$ and $Q^{2}=2 \mathrm{GeV}^{2}$ to $109 \mathrm{GeV}^{2}$. See text for details.

the fragmentation functions terminate at $z_{2}=1 /(1+r)$ as appropriate. The intial condition, which is merely the product of two single fragmentation functions and is not subjected to this constraint, does not show this behaviour. It is imposed by hand, in the initial condition, that they vanish at and above this value.

In experiments, one can first identify a hadron as the leading hadron inside a jet and use it as a trigger with given momentum $z_{1}$. Then, the associated or the "nextleading" hadron distribution inside the same jet corresponds to the ratio of dihadron and single hadron fragmentation functions, $D^{h_{1}, h_{2}}\left(z_{1}, z_{2}\right) / D^{h_{1}}\left(z_{1}\right)$. We present results for this ratio at $z_{1}=2 z_{2}$ in Fig. 15 at $z_{1}=3 z_{2}$ in Fig. 16 and in the extreme cases of $z_{1}=z_{2}$ in Fig. 17 and $z_{1}=4 z_{2}$ in Fig. I8 It should be pointed out that the $y$-axis in all these plots is linear and not logarithmic. Thus, one concludes that the ratio demonstrates little qualitative change for a variation of $Q^{2}$ by almost two orders of magnitude. In making such an observation, one must ignore the difference between the solid line (initial condition) and the remaining lines (evolved functions) especially at large $z_{2}$. This is due to the fact that the initial condition is not subjected to the kinematic constraint as $z_{2} \rightarrow 1 /(1+r)$. However, the four plots are visually quite different from each other: the ratios of the evolved functions display a steady drop as compared to the ini- 


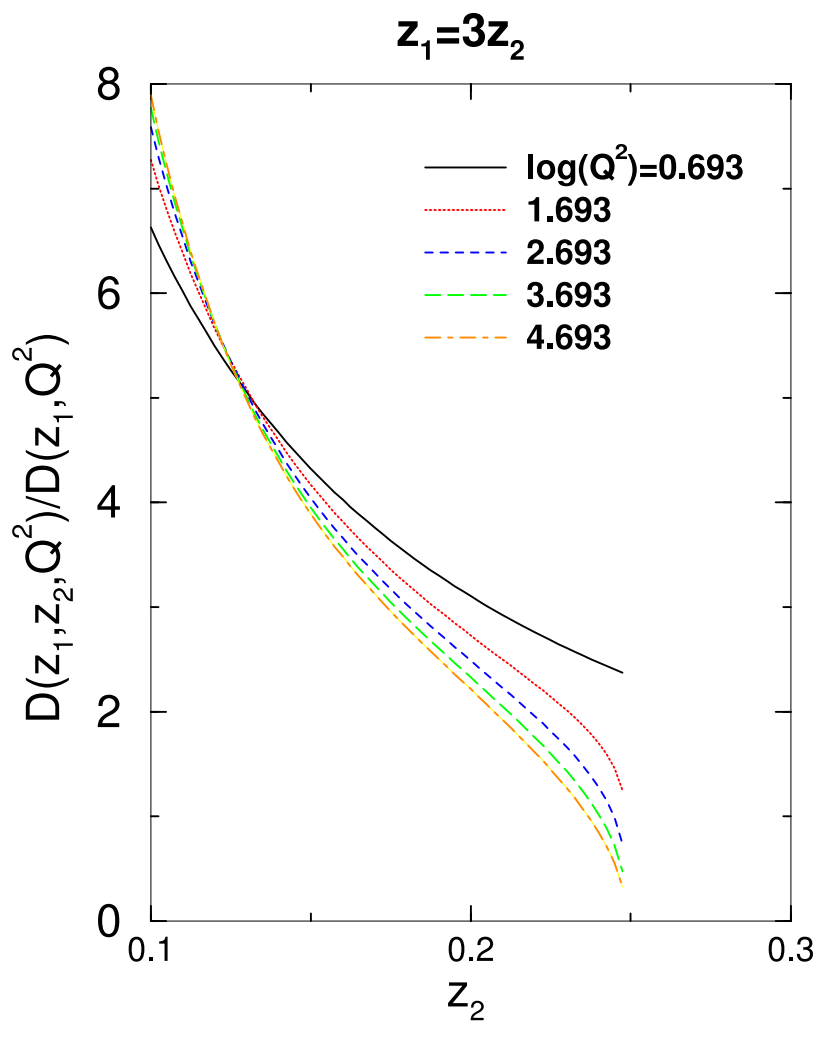

FIG. 16: Same as Fig. 15 except $z_{1}=3 z_{2}$.

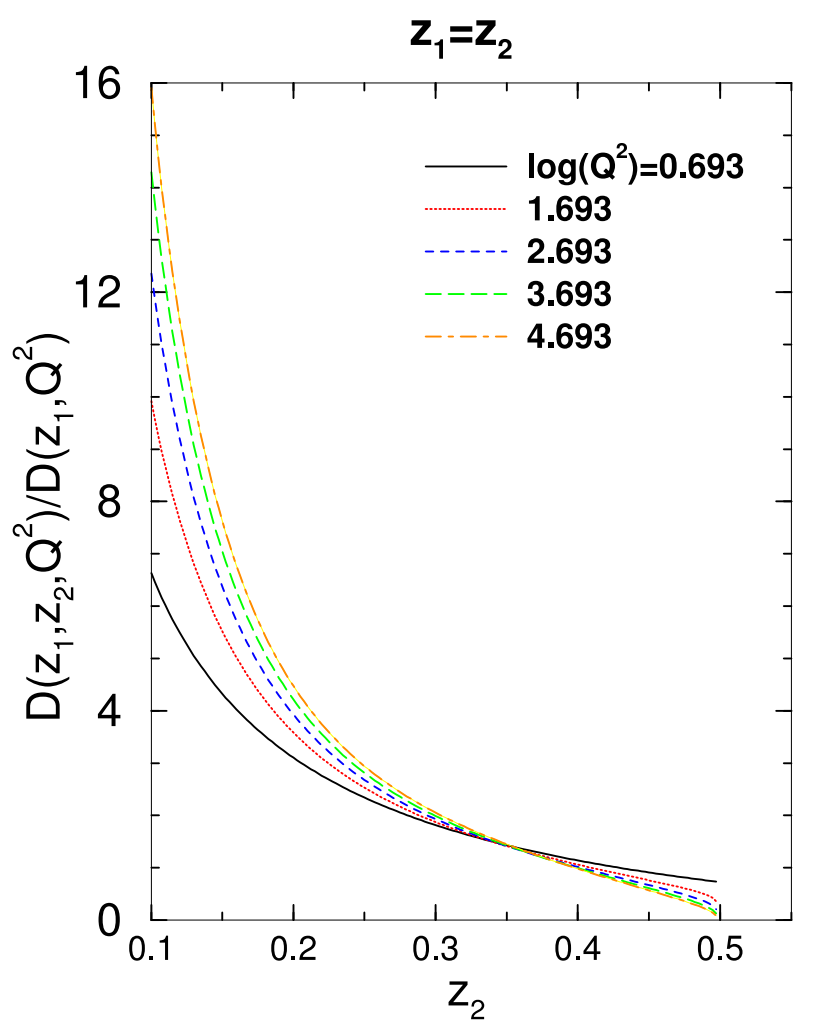

FIG. 17: Same as Fig. 15 except $z_{1}=z_{2}$. tial condition as we progress from $z_{1}=z_{2}$ to $z_{1}=4 z_{2}$. The reader will also note that the maximum of the $y$-axis drops with increasing $r$.

As the energy of the $e^{+} e^{-}$annihilation is raised the multiplicity must also increase. The cause for this is nothing other than the excess energy available for particle production. Following the physical picture of fragmentation proposed in Ref. 22 , one notes that at higher energies it becomes more probable that the two hadrons emanate from two causally disconnected sectors of the fragmenting jet. If this were the case, then at very high energies, the ratio of the double fragmentation function to the product of the single fragmentation functions $\left(D_{q}^{h_{1} h_{2}}\left(z_{1}, z_{2}, Q^{2}\right) / D_{q}^{h_{1}}\left(z_{1}, Q^{2}\right) D_{q}^{h_{1}}\left(z_{2}, Q^{2}\right)\right)$ should reach unity, especially for small values of $z_{1}, z_{2}$. This has not turned out to be the case as evidenced by the plots of this ratio in Fig. 19 for $z_{1}=2 z_{2}$ and in Fig. 201 for $z_{1}=3 z_{2}$. In these plots the ratio deviates from unity at small momentum fractions. Whether this is a facet of the choice of our initial conditions is as yet unclear.

\section{DISCUSSIONS AND CONCLUSIONS}

In this paper, we have studied dihadron fragmentation functions within the framework of collinear factorization in the high-energy $e^{+} e^{-}$annihilation processes, starting with the operator definition. Using the cut-vertex tech- nique, we also derived the DGLAP evolution equation for the non-singlet dihadron quark fragmentation function. We solved the DGLAP evolution equation numerically, with a simple ansatz for the initial condition.

Both the definition in operator formalism and the resultant DGLAP evolution equations for the dihadron fragmentation functions have remarkable similarities to the single fragmentation functions. The first type of contribution to the evolution equations, that from gluon radiation before the fragmentation of the offspring quark (or gluon) into a pair of hadrons, is very similar to the corresponding process in single fragmentation functions. The second type, unique to the dihadron fragmentation functions, comes from independent fragmentation of the two offspring partons into two single hadrons of the observed pair. Since this piece represents the incoherent fragmentation of the quark and the gluon, it is well defined only when the transverse momentum between the detected hadrons is large.

The relative transverse momentum between the two hadrons is integrated over in the definition of the dihadron fragmentation function. Hence, we have assumed that its non-perturbative contribution, which resides in the operator definition, is restricted to an intrinsic transverse momentum scale, $q_{\perp}<\mu_{\perp}$. Hadron pairs with $q_{\perp}>\mu_{\perp}$ are assumed to be generated only perturbatively. For this assumption to be justified, the semihard scale $\mu_{\perp}$ is chosen to be much larger than $\lambda_{Q C D}$. One has 


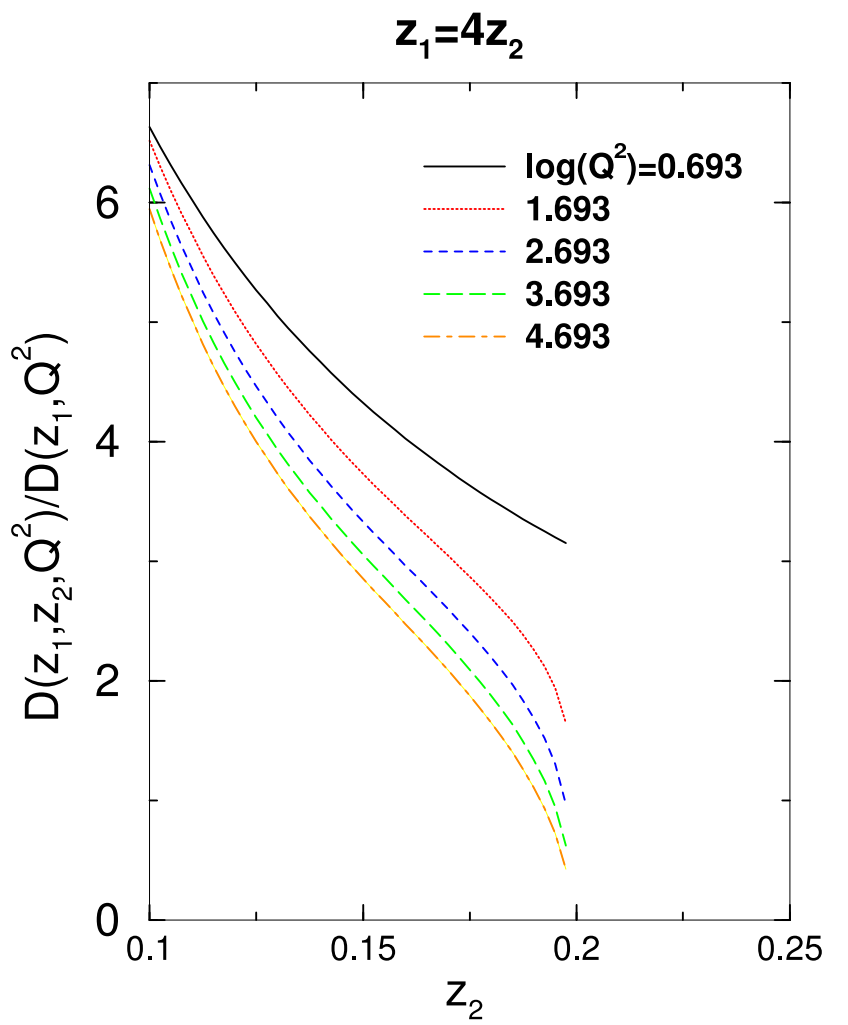

FIG. 18: Same as Fig. 15 except $z_{1}=4 z_{2}$.

then to assume that the energy scales of the processes in question i.e. $Q^{2}$ be much larger than the semihard scale $\mu_{\perp}$, such that a hierarchy of scales, $\Lambda_{Q C D}^{2}<<\mu_{\perp}^{2}<<$ $Q^{2}$, is satisfied.

This study is motivated by the observation 4 that the same side correlations of two high $p_{T}$ hadrons in central $A u+A u$ collisions remain approximately unchanged as compared with that in $p+p$ and $d+A u$ collisions. Specifically in this experiment, one measures the distribution, in azimuthal angle, of the secondary (or associated) hadron $\frac{1}{N_{\text {trig }}} \frac{d N}{d \phi}$ with respect to the triggered high $p_{T}$ hadron. Neglecting the differences in production cross section and fragmentation functions for different parton species, the integrated yield of the correlation around the peak at $\phi=0$ should be the ratio of dihadron and single hadron fragmentation functions, $D_{a}^{h_{1} h_{2}}\left(z_{1}, z_{2}, Q^{2}\right) / D_{a}^{h_{1}}\left(z_{1}, Q^{2}\right)$, with $z_{1}$ and $z_{2}$ being the momentum fractions of the triggered hadron and associated hadrons, respectively. To understand the observation in the framework of jet quenching, one has to study the medium modification to a dihadron fragmentation function due to parton energy loss. Since it has been shown in the case of single fragmentation functions that medium modification due to multiple scattering and induced gluon radiation closely resemble that of radiative corrections due to evolution in vacuum 17, the DGLAP evolution is expected to yield clues regarding the medium modification of the dihadron fragmentation functions.

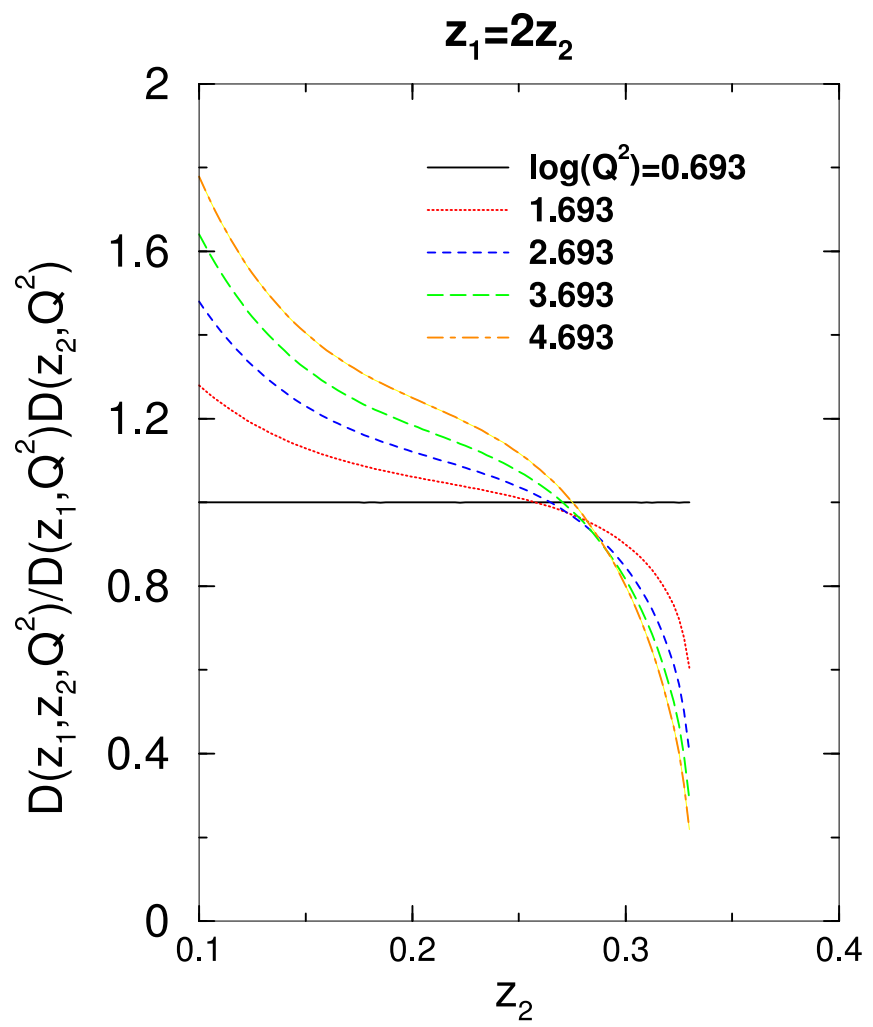

FIG. 19: Results of the ratio of the non-singlet quark dihadron fragmention function $D_{q}^{h_{1} h_{2}}\left(z_{1}, z_{2}, Q^{2}\right)$ to the product of the single fragmentation functions $D_{q}^{h_{1}}\left(z_{1}, Q^{2}\right) D_{q}^{h_{1}}\left(z_{2}, Q^{2}\right)$. In this case $z_{1}=2 z_{2}$ and $Q^{2}=2 \mathrm{GeV}^{2}$ to $109 \mathrm{GeV}^{2}$. See text for details.

Our numerical results indeed show little change of the ratio $D_{q}^{h_{1} h_{2}}\left(z_{1}, z_{2}, Q^{2}\right) / D_{q}^{h_{1}}\left(z_{1}, Q^{2}\right)$ as $Q^{2}$ is varied in a wide range of values. The evolution is shown to be strongly dependent, however, on the ratio of the momentum fractions of the two hadrons $\left(r=z_{1} / z_{2}\right)$. In the results of Ref. $A$ the ratio $r=z_{1} / z_{2}$ is essentially integrated over all values $\geq 1$. In order to relate to the results in this paper one essentially must average the effects of evolution shown in Figs. 1518 . One will immediately note that summing over different values of the ratio $z_{1} / z_{2}$ will lead to the observation of minimal change in the ratio of the fragmentation functions as a function of the $Q^{2}$ of the reaction.

No doubt, this study is but the first step in this effort. In the interest of simplicity, results for the computationally simpler non-singlet fragmentation functions were presented. The results for the evolution of the more physically relavant singlet fragmentation functions will be presented in a future effort. The DGLAP evolution equations for such functions will involve, in addition, the splitting of one gluon to two gluons and the coupled differential equations.

In the above, we have demonstrated the factorization of the double differential cross section into a LO hard part and a soft piece which encoded the nonperturbative infor- 


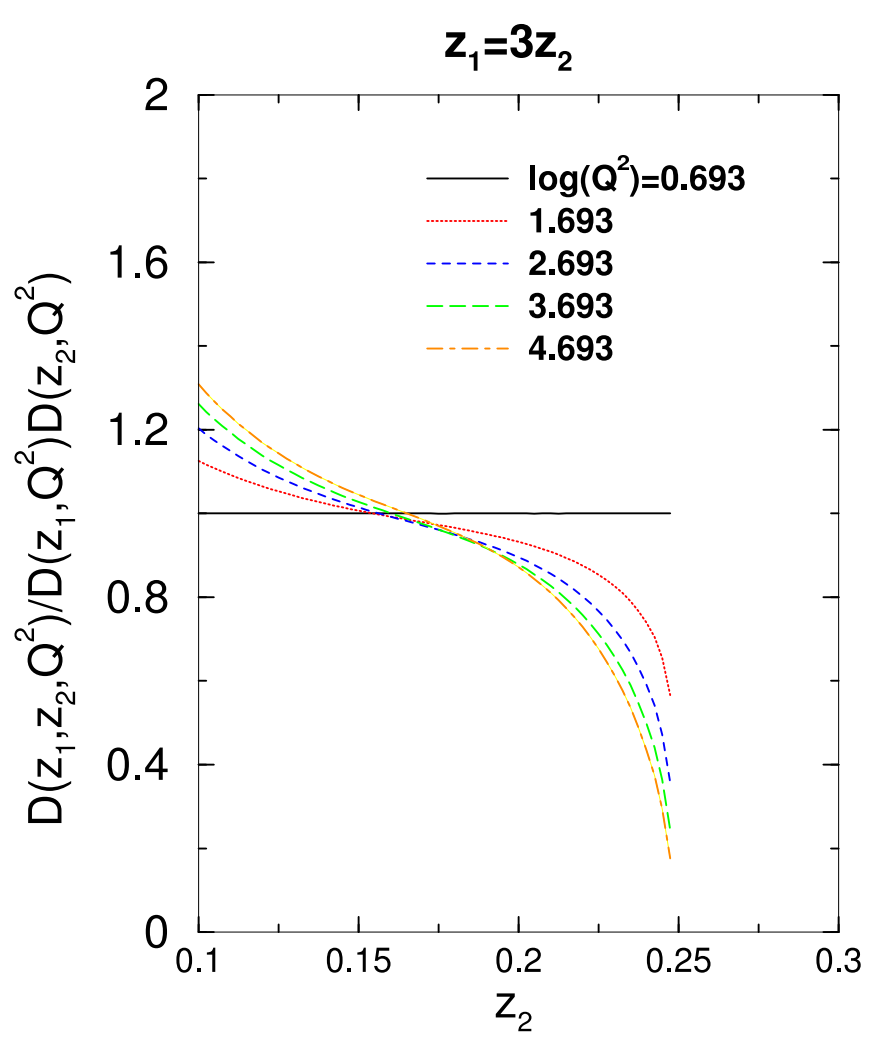

FIG. 20: Same as Fig. [9 except $z_{1}=3 z_{2}$

mation of converting partons into hadrons. A complete proof of factorization requires the extension of the calculation to all orders. There also remains the evaluation of the medium modifications to the dihadron fragmentation functions. We will address each of these issues, in turn, in future publications.

\section{Acknowledgments}

The authors wish to thank V. Koch, J. Qiu and G. Sterman for helpful discussions. This work was supported in part by the Natural Sciences and Engineering Research Council of Canada, and in part by the Director, Office of Science, Office of High Energy and Nuclear Physics, Division of Nuclear Physics, and by the Office of Basic Energy Sciences, Division of Nuclear Sciences, of the U.S. Department of Energy under Contract No. DE-AC03-76SF00098.

\section{APPENDIX A: SUDAKOV DOUBLE LOGARITHMS IN LIGHT-CONE GAUGE}

In Subsect. c of Sect. IV we argued that one of the NLO contributions to the dihadron fragmentation functions would result from the convolution of two single fragmentation functions. The justification for the inclusion of this process rests on the assumption that the higher order diagrams that have gluon lines connecting the outgoing quark and gluon can be ignored in a leading log analysis. The simplest higher order correction of this type emanates from the presence of a single gluon line connecting the outgoing quark and gluon as illustrated in Fig. 211

The presence of a Sudakov double logarithm in this diagram signals a leading log contribution to the infrared and collinear divergences of diagrams of the same order. Such a contribution will call into question our derivation of the second piece of the evolution equation [Eq. [74]. Derivation of this piece of the evolution equations required the identification of the leading log contributions from infrared and collinear divergences followed by factorization and a resummation of these into the single fragmention functions. Such a procedure may only be carried out if the leading log portions in higher order diagrams are contained solely in the selfenergy corrections of the outgoing gluon and quark lines and in real gluon or quark emmissions off these lines. A leading log contribution from the infrared or collinear sector of the diagram of Fig. 2ll will call this procedure into question. In this appendix, we demonstrate that in the collinear sector of this diagram, evaluated in light-cone gauge, the Sudakov double log is absent.

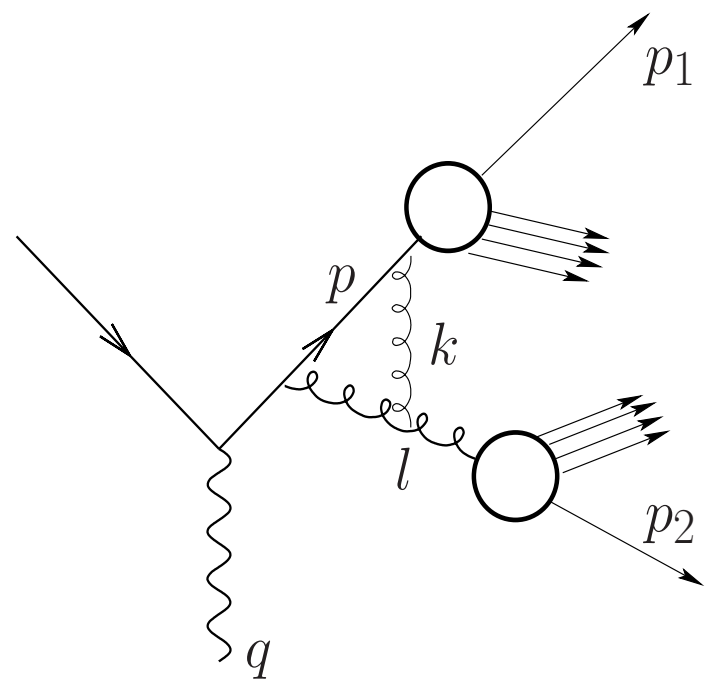

FIG. 21: A next-to-next-to-leading order correction.

In this endevour we follow the techniques outlined in Ref. 8 for the evaluation of the photon vertex. The leading contributions from the diagram of Fig. 21 may be evaluated as in $\delta$ by the solution of the Landau equations. This leads to the presence of two regions of phase space where a double logarithm may arise: 


$$
\begin{array}{cl}
p^{\mu}+k^{\mu}=\alpha_{1} k^{\mu} & , \mathbf{k}^{2}=\mathbf{p} \cdot \mathbf{k} \\
l^{\mu}-k^{\mu}=\alpha_{2} k^{\mu} & , \mathbf{k}^{2}=\mathbf{p} \cdot \mathbf{k}
\end{array}
$$

In the above, $\alpha_{1}, \alpha_{2}$ are mere real numbers. These conditions essentially outline the cases where the gluon $k^{\mu}$ achieves collinearity with the outgoing quark with momentum $p^{\mu}$ or with the outgoing gluon with momentum $l^{\mu}$. Either case produces a double log contribution in the Feynman gauge.

The expression for a quark with momentum $\mathbf{p}+\mathbf{l}$ splitting into an outgoing onshell quark with momentum $\mathbf{p}$ and gluon with momentum $\mathbf{l}$ with the splitting vertex corrected by a single gluon with momentum $\mathbf{k}$ may be expressed as

$$
\begin{aligned}
& \bar{u}_{i}(p) \Gamma_{\rho_{i, j}}^{a} u_{j}(p+l) \varepsilon_{a}^{\rho}(l)=\bar{u}_{i}(p) \int \frac{d^{4} k}{(2 \pi)^{4}} i t^{d} g \gamma^{\nu} \\
& \times \frac{i(\not \mathbf{p}+\mathbf{k})}{(\mathbf{p}+\mathbf{k})^{2}+i \epsilon} i t^{c} g \gamma^{\mu} \frac{i d_{\alpha \mu}(\mathbf{l}-\mathbf{k})}{(\mathbf{l}+\mathbf{k})^{2}+i \epsilon} g f^{a c d} \\
& \quad \times\left[g^{\rho \alpha}(k-2 l)^{\beta}+g^{\alpha \beta}(l-2 k)^{\rho}+g^{\beta \rho}(k+l)^{\alpha}\right] \\
& \quad \times \frac{i d_{\beta \nu}(k)}{\mathbf{k}^{2}+i \epsilon} \varepsilon_{p}^{a *}(l) .
\end{aligned}
$$

It may be argued (see Ref. 8 ), that the double logarithm behaviour in vertex diagrams originates on the collinear pinch surfaces outlined above in Eq. A1]. The behaviour of this above vertex correction in the region where the internal gluon momentum $\mathbf{k}$ becomes collinear to the the outgoing gluon momentum $\mathbf{l}$ will now be analysed. This corresponds to the second condition in Eq. A1. The $d^{4} k$ integration is decomposed into the light-cone variables $d k^{+} d k^{-} d^{2} k_{\perp}$. The focus is on the pinch sigularity which results from the two denominators

$$
\frac{1}{\mathbf{k}^{2}+i \epsilon}, \frac{1}{(\mathbf{l}-\mathbf{k})^{2}+i \epsilon}
$$

The pinch between the two denominators arises in the $k^{-}$integration solely in the region $0<k^{+}<l^{+}$. Evaluating the pole at $k^{-}=k_{\perp}^{2} / 2 k^{+}$. We obtain a pinch from the

$$
\frac{1}{(\mathbf{l}-\mathbf{k})^{2}+i \epsilon}
$$

propagator in the region where $\vec{k}_{\perp} \cdot \vec{l}_{\perp}=k_{\perp} l_{\perp}$, i.e. $\mathbf{k}$ becomes collinear with $\mathbf{l}$. To regulate the collinear divergence we introduce the variables $x, \delta \vec{k}_{\perp}$ :

$$
\begin{aligned}
& k^{+}=x l^{+} \\
& \vec{k}_{\perp}=x \vec{l}_{\perp}+\delta \vec{k}_{\perp} .
\end{aligned}
$$

Evaluating the integrand of Eq. $\mathbf{A}$ ? at the residue of the pole $k^{-}=k^{2} / 2 k^{+}$followed by the substitutions outlined in Eq. $\quad$ A3 we obtain the split vertex correction as

$$
\begin{array}{ll}
\bar{u}_{i}(p) \Gamma_{\rho_{i, j}}^{a} u_{j}(p+l) \varepsilon_{a}^{\rho}(l)=S^{a} \int \frac{d x}{x} \int \frac{d^{2} \delta k_{\perp}}{\delta k_{\perp}^{2}} \\
\times \quad \bar{u}(p) \gamma^{\nu} \frac{(\mathbf{p}+x y) p^{+} l^{+}}{l_{\perp}^{2}\left(p^{+}+l^{+}\right)^{2}} \gamma^{\mu} u(p+l) \\
\times \quad\left[-g_{\alpha \mu}+\frac{(1-x)\left(l_{\alpha} n_{\mu}+l_{\mu} n_{\alpha}\right)}{(1-x) \mathbf{l} \cdot \mathbf{n}}\right] \\
\times \quad\left[g^{\rho \alpha}(x-2) l^{\beta}+g^{\alpha \beta}(1-2 x) l^{\rho}+g^{\beta \rho}(x+1) l^{\alpha}\right] \\
\times \quad d_{\beta \nu}(l) \varepsilon_{p}^{a *}(l) .
\end{array}
$$

In the above equation all factors of color together with multiplicative constants have been absorbed into the factor $S^{\alpha}$. The sum over polarizations of the gluon with momentum $\mathbf{l}-\mathbf{k}\left[\right.$ i.e. the factor $d_{\alpha \mu}(\mathbf{l}-\mathbf{k})$ ] and the expression for the glue vertex have been simplified. In these and in the rest of the numerator, factors of $\delta \vec{k}_{\perp}$ have been neglected, as the focus is on the region where $\delta k_{\perp} \rightarrow 0$. If the numerator, under this approximation, turned out to be vanishing, then this would indicate the leading contribution to be proportional to $\delta k_{\perp} \rightarrow 0$ and as a result no double logarithm and no leading contribution from this loop would result. Under the relations afforded by the polarizations of the light-cone gauge:

$$
l^{\beta} \cdot d_{\mu \beta}=0, \quad l^{\rho} \cdot \varepsilon_{\rho}^{a *}(l)=0,
$$

it may be easily demonstrated that a contraction of the Lorentz indices $\alpha, \rho, \beta$ lead to the numerator of the r.h.s. of Eq. A4 to become vanishing. This result is a property of the light-cone gauge.

It may also be demonstrated, following similar methods, that the double logarithm emanating from the region of phase space where the gluon $k^{\mu}$ becomes collinear with the outgoing quark line is also suppressed due the vanishing of its coefficient as above. We leave the proof of this property to the reader. The above arguments demonstrate the vanishing of the leading contributions form higher order corrections to the split vertex. This property validates our picture of independent fragmentation. 
[1] For a review see F. Karsch and E. Laermann, arXiv hep-lat/0305025

[2] M. Gyulassy and M. Plumer, Phys. Lett. B 243, 432 (1990); X. N. Wang and M. Gyulassy, Phys. Rev. Lett. 68, $1480(1992)$.

[3] K. Adcox et al. [PHENIX Collaboration], Phys. Rev. Lett. 88, 022301 (2002) [arXiv nucl-ex/0109003; C. Adler et al. [STAR Collaboration], Phys. Rev. Lett. 89, 202301 (2002) [arXiv nucl-ex/0206011.

[4] C. Adler et al. [STAR Collaboration|, Phys. Rev. Lett. 90, 082302 (2003) [arXiv nucl-ex/0210033.

[5] G. Sterman, and S. Weinberg, Phys. Rev. Lett. 39, 1436 (1977).

[6] Yu. L. Dokshitser, Zh. Eksp. Teor. Fiz. 73, 1216 (1977).

[7] R. D. Feild, and R. P. Feynman, Nucl. Phys. B136, 1, (1978).

[8] J. C. Collins, D. E. Soper, and G. Sterman, in Perturbative Quantum Chromodynamics edited by A. Mueller, (World Scientific 1989), and references therein.

[9] A. H. Mueller, Phys. Rev. D. 18, 3705 (1978).

[10] V. N. Gribov, and L. N. Lipatov, Sov. J. Nucl. Phys. 15, 438 (1972).

[11] Yu. L. Dokshitzer, Sov. Phys. JETP 46, 641 (1977).

[12] G. Altarelli, and G. Parisi, Nucl. Phys. B126, 298 (1977).

[13] K. Konishi, A. Ukawa, and G. Veneziano, Phys. lett. 78B, 243 (1978).
[14] K. Konishi, A. Ukawa, and G. Veneziano, Phys. lett. 80B, 259 (1979).

[15] K. Konishi, A. Ukawa, and G. Veneziano, Nucl. Phys. B157, 45 (1979).

[16] U. P. Sukhatme, and K. E. Lassila, Phys. Rev. D. 22, 1184 (1980).

[17] X. F. Guo and X. N. Wang, Phys. Rev. Lett. 85, 3591 (2000) [arXiv hep-ph/0005044; X. N. Wang and X. F. Guo, Nucl. Phys. A 696, 788 (2001) [arXiv hep-ph/0102230 .

[18] R. D. Field, Applications of Perturbative QCD, AddisonWesley, New York (1995).

[19] R. Akers et al. [OPAL Collaboration], Z. Phys. C 63, 197 (1994).

[20] A. Mueller, Phys. Rept. 73, 237 (1981).

$\left[21^{\circ}\right.$ J. Osborne, E. Wang and X. N. Wang, Phys. Rev. D. 67, 094022 (2003).

[22] Yu. L. Dokshitser, V. A. Khoze and S. I. Troyan, in Perturbative Quantum Chromodynamics edited by A. Mueller, (World Scientific 1989), and references therein.

[23] T. Muta, Foundations of Quantum Chromodynamics, World Scientific, Singapore, (1998).

[24] J. C. Collins and J. Qiu, Phys. Rev. D. 39, 1398 (1989).

[25] C. Itzykson, J. B. Zuber, Quantum Field Theory, McGraw Hill, New York, (1980). 